\title{
Neuronal behaviors: a control perspective
}

\author{
Guillaume Drion, Timothy O’Leary, Julie Dethier, Alessio Franci, Rodolphe Sepulchre
}

\begin{abstract}
The purpose of this tutorial is to introduce and analyze models of neurons from a control perspective and to show how recently developed analytical tools help to address important biological questions. A first objective is to review the basic modeling principles of neurophysiology in which neurons are modeled as equivalent nonlinear electrical circuits that capture their excitable properties. The specific architecture of the models is key to the tractability of their analysis: in spite of their high-dimensional and nonlinear nature, the model properties can be understood in terms of few canonical positive and negative feedback motifs localized in distinct timescales. We use this insight to shed light on a key problem in experimental neurophysiology, the challenge of understanding the sensitivity of neuronal behaviors to underlying parameters in empiricallyderived models. Finally, we show how sensitivity analysis of neuronal excitability relates to robustness and regulation of neuronal behaviors.
\end{abstract}

\section{INTRODUCTION}

Models of neurons have long been a fruitful source of inspiration in physics and engineering and have revolutionized our understanding of nervous system function. Within neuroscience, models of neurons and neuronal networks provide a means of mechanistically and quantitatively understanding nervous system function. In related fields these models have inspired the development of machine learning, a most successful branch of engineering intimately connected to computational neuroscience, as well as many recent developments in dynamical systems theory that are intimately connected to mathematical neuroscience. Surprisingly, neuroscience and control theory have evolved quite separately, in spite of their common roots in cybernetics and their shared terminology of circuit theory. It is therefore timely to redress this divergence between disciplines and regard models of neurons as behaviors [43], that is, open systems amenable

G. Drion and J. Dethier are with the Department of Electrical Engineering and Computer Science, University of Liège, 4000 Liège, Belgium, gdrion@ulg.ac.be, jdethier@ulg.ac.be. T. O'Leary and G. Drion are with Brandeis University, Volen Center for Complex Systems, Mailstop 013, 415 South Street, Waltham MA 02454, USA, tolearyebrandeis.edu Alessio Franci is with the National Autonomous University of Mexico, Science Faculty, Department of Mathematics, Av. Universidad 3000, Coyoacán, 04510 Ciudad Universitaria, D.F. México afrancieciencias.unam.mx R. Sepulchre is with the University of Cambridge, Department of Engineering, Trumpington Street, Cambridge CB2 1PZ, and with the Department of Electrical Engineering and Computer Science, University of Liège, 4000 Liège, Belgium, r.sepulchreeeng.cam.ac.uk.

This paper presents research results of the Belgian Network DYSCO (Dynamical Systems, Control, and Optimization), funded by the Interuniversity Attraction Poles Programme, initiated by the Belgian State, Science Policy Office. G.D. is a Marie-Curie COFUND postdoctoral fellow at the University of Liege. Co-funded by the European Union. J.D. is supported by the F.R.S.-FNRS (Belgian Fund for Scientific Research. The scientific responsibility rests with its authors. to interconnection, tearing, and zooming, and, above all, regulated by feedback principles.

The focus of this paper is a threefold message: (i) neuronal models are nonlinear electrical circuits; as such, they fit the primary language of behavioral theory prior to being regarded as high-dimensional sets of nonlinear differential equations; (ii) neuronal models are excitable behaviors; as such, they offer a unique modeling substrate for the behavioral treatment of a system property that is fundamental to biology; (iii) the mechanisms of regulation, homeostasis, robustness, modulation, and sensitivity of neuronal behaviors underlie key questions of current experimental neurophysiology; as such, they match the core questions of control theory but the lack of answers calls for novel analysis tools tailored to the excitable nature of neuronal behaviors.

An emphasis of this tutorial paper is on the value of studying neuronal models at a time of growing interest in the control community for systems and synthetic biology. At first sight, studying the electrical activity of a single excitable cell may appear old-fashioned in the age of molecular biology. However, while molecular biology has provided unprecedented scope for studying and manipulating biological systems, this has perhaps come at the cost of focussing on dynamics, or, in biological terminology, physiology. Control theory is to a large extent model-based, and the landmark modeling paper of Hodgkin and Huxley in 1952 [23] represents an unparalleled success story of biological modeling in the 20th century. The biophysical modeling principles of this paper are mostly unchallenged to date and have provided a solid foundation for sixty years of experimental neurophysiology and computational modeling. Moreover, those principles rely on circuit principles quite familiar to control engineers, which reduces the terminological gap between control theorists and experimental neurophysiologists. For this reason, the conductance-based models of neurophysiology offer a specific yet rich window to the investigation of model-based biological behaviors and it is our belief that many of the principles of neuronal behaviors are quite general in biology.

The research questions that underlie the model-based investigation of neuronal behaviors share many common features with research questions that nowadays attract control theorists in systems biology and synthetic biology. Neuronal behaviors are biological behaviors of general interest in that they are excitable behaviors regulated across many scales. As a behavioral property, excitability is found across biology, and could even be argued to be one of the few defining properties of living organisms. Whereas excitability modeling is relatively scarce in most biological contexts 
because of the paucity of reliable spatio-temporal data, conductance-based models are broadly accepted as adequate mathematical models of neuronal excitability both qualitatively and quantitatively. Conductance based models also provide a milestone in the modeling of behaviors across scales. As the interconnection between an electrical circuit at the cellular scale and a biochemical reaction network at the molecular scale, conductance based models offer a unique paradigm for the modeling, analysis, and synthesis of behaviors organized at a coarser scale by the collective organization of a population at a finer scale. The need to understand biology across scales, from the smallest identifiable components to whole organisms, was what motivated the investigation of neuronal networks in the first place, but most of the initial questions remain largely open and relevant beyond the particular context of single-cell neuronal activity.

The organization of the paper is as follows. Section II revisits the modeling principles of conductance-based neuronal models, with an emphasis on the model as the interconnection of an electrical circuit with a biochemical network. Section III shows that the modulation of positive and negative feedback in a nonlinear resistive circuit is a basic mechanism for the regulation of excitable behaviors. Section IV highlights the importance of local sensitivity analysis in experimental and computational neurophysiology. Finally, sections V and VI propose new methodologies for the analysis of sensitivity, robustness and regulation of neuron conductance-based models.

\section{THE HERITAge OF HodgKIN AND HuXLEY}

\section{A. Neuronal models are electrical circuits}

The seminal paper [23] concludes a series of papers that led the biophysical foundations of modern neuroscience.The first figure of the paper, reproduced in Figure 1A is the representation of the neuronal model as an RC electrical circuit. Neurons have a membrane that can maintain a voltage difference between the extracellular and intracellular medium. The cellular membrane is therefore modeled as a capacitor. The voltage across the membrane can vary because ions (primarily sodium $\left(\mathrm{Na}^{+}\right)$, potassium $\left(\mathrm{K}^{+}\right)$, and calcium $\left(\mathrm{Ca}^{2+}\right)$ ) can flow through the membrane via specific transmembrane proteins called ion channels (Figure 2A). Each ionic current is modeled as a resistor in series with a battery. The battery represents the equilibrium (Nernst) potential at which there is no flow. This equilibrium potential is higher than the resting potential of the neuron for sodium and calcium ions. It is lower than the resting potential for potassium ions. Therefore an inward flow of sodium or calcium depolarizes the membrane voltage, whereas an (outward) flow of potassium is hyperpolarizes the membrane voltage (Fig. 2B).

1) Circuit equation: Hodgkin-Huxley model only includes two ionic currents: a depolarizing sodium current and an hyperpolarizing potassium current (calcium channels were discovered soon after their seminal work). The remaining currents are lumped in a passive leakage current. The circuit equations of Hodgkin-Huxley model are readily derived from the circuit diagram. Kirchhoff's law states that all currents must sum to zero, that is

$$
C \frac{d V}{d t}=I_{N a}+I_{K}+I_{l}+I_{a p p}
$$

where $I_{a p p}$ is an externally applied current. Ohm's law states that each ionic current is proportional to the voltage deviation from its equilibrium potential:

$$
\begin{array}{rll}
I_{N a}(t) & = & g_{N a}(t)\left(V(t)-E_{N a}\right) \\
I_{K}(t) & =g_{K}(t)\left(V(t)-E_{K}\right) \\
I_{l} \quad(t)= & g_{l}\left(V(t)-E_{l}\right)
\end{array}
$$

A fundamental contribution of Hodgkin and Huxley was to separate experimentally the contribution of sodium and potassium currents in order to model the voltage dependence of their respective conductances. The voltage dependence of ionic conductances is an essential source of nonlinearity of the model.

Today's models of neurons are build upon the same circuit principles as in the original Hodgkin-Huxley paper. They often contain many more ionic branches because each ionic current can flow in many different ways in a specific neuron and much progress has been achieved in the quantitative modeling of those conductances (see next section). Figure 1B illustrates the circuit equivalent of a recent model of midbrain dopaminergic neurons. The model contains two distinct compartments accounting for different parts of the neuron: the soma, or cell body; and dendrites, thin structures that arise from the cell body and receive most of the inputs from efferent neurons. These structures are distinct in the model because ionic flows are usually different near the soma and in dendrites. Each compartment contains several distinct ionic currents. The soma compartment model in Figure 1.B contains three different types of calcium currents and three different types of potassium currents. All those currents obey an ohmic law, like in Hodgkin-Huxley model, but they differ in their conductance because they correspond to different types of ion channels. The switch indicated in the ionic branch of $I_{K, S K}$ is because the paper [5] studies among other things the behavior effect of specifically blocking this $S K$ current with a specific drug.

2) Internal and external currents: The sodium, potassium, and calcium currents so far discussed are internal currents: their conductances only depend on the neuron variables (primarily its voltage potential, and possibly ionic intracellular concentrations). In contrast, the currents $I_{G A B A, A}$, $I_{A M P A}$, and $I_{N M D A}$ are external synaptic currents (the terms $G A B A, A, A M P A$ and $N M D A$ account for different types of membrane receptors having specific dynamics). Those currents result from synaptic interconnections with other neurons. Their conductances will therefore depend on external variables, such as the membrane potentials of connected neurons or the concentration of various external chemical compounds that are released by the efferent cells, called neurotransmitters. The switches in the corresponding branches indicate that such currents can be turned on and off by neurotransmitters. The circuit modeling principles do not 


\section{A Hodgkin-Huxley circuit}

(Hodgkin and Huxley, 1952)

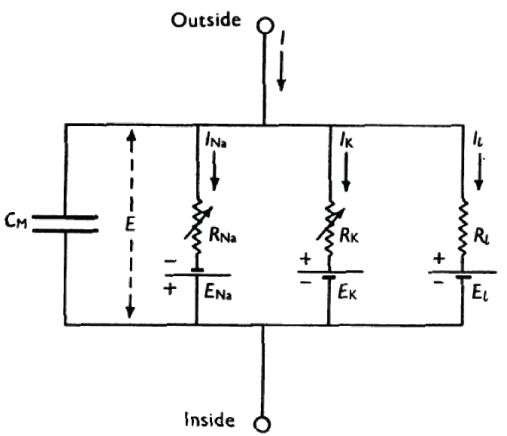

B A "modern" circuit

(Canavier and Landry, 2006)

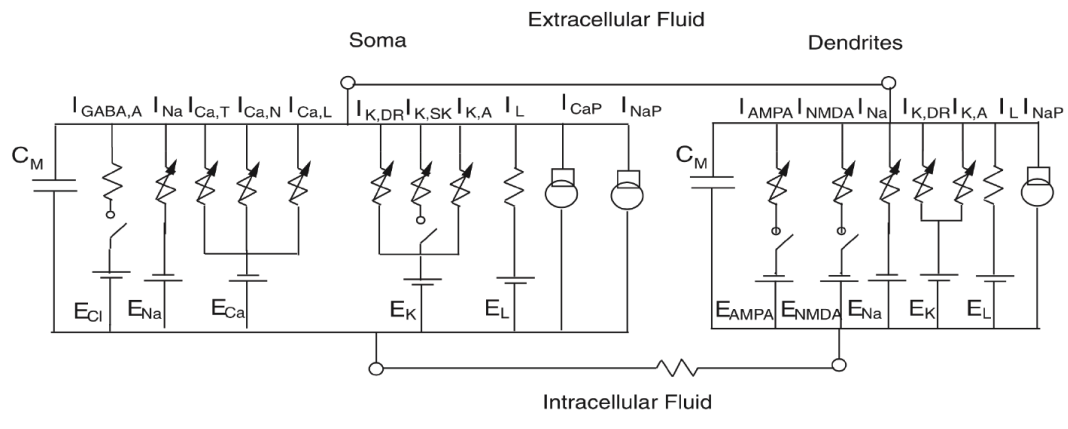

Fig. 1. Examples of neuronal circuits. A. Electrical circuit of the Hodgkin Huxley model (taken from [23]). B. Modern electrical circuit of a dopaminergic neuron model (taken from [5]).

differ for internal or external currents, but external currents are not further considered in the present paper, which for simplicity focuses on the endogenous behavior of a single cell.

The pump currents $I_{N a, P}$ and $I_{C a, P}$ are included in Figure $1 \mathrm{~B}$ because a model that includes the dependence of conductances in the calcium and sodium intracellular concentration must include those concentrations as state variables. The behavior of those concentrations is modeled by a balance between the corresponding ionic currents and pump currents, that biochemically restore the equilibrium concentrations. The simplest electrical analog of a pump current is a current source (statically) dependent on the ion concentration.

The important take-home message of this short section is that neuronal models are electrical circuits before anything else. Each neuron is primarily a one-port device and Kirchhoff's law provides a scalar equation that describes its I-V behavior. It could be added that the flow of the capacitive current is so fast with respect to all active ionic flows that its dynamics can be safely neglected. From an electrical viewpoint, a neuronal model can therefore be thought of as a purely (nonlinear and time-dependent) resistive circuit. In spite of their nonlinear dynamics, neuronal behaviors satisfy Kirchhoff's laws at any instant of time, meaning that the ionic currents flowing through the cellular membrane always sum to zero.

\section{B. Conductances model the cellular correlate of molecular biochemical processes}

1) The voltage-clamp experiment: Most of the experimental work of Hodgkin and Huxley went into modeling the sodium and potassium conductances in the squid axon, taking advantage of its large ( $\mathrm{mm}$ scale) dimensions. The breakthrough came from a novel experimental technique, the voltage clamp experiment. The experiment uses a feedback amplifier (the novel "hidden technology" of the time) to control the steady-state value of the membrane potential. Neglecting the (fast) capacitive current in the circuit equation (1), the current change $\Delta I(t)$ required to control a voltage

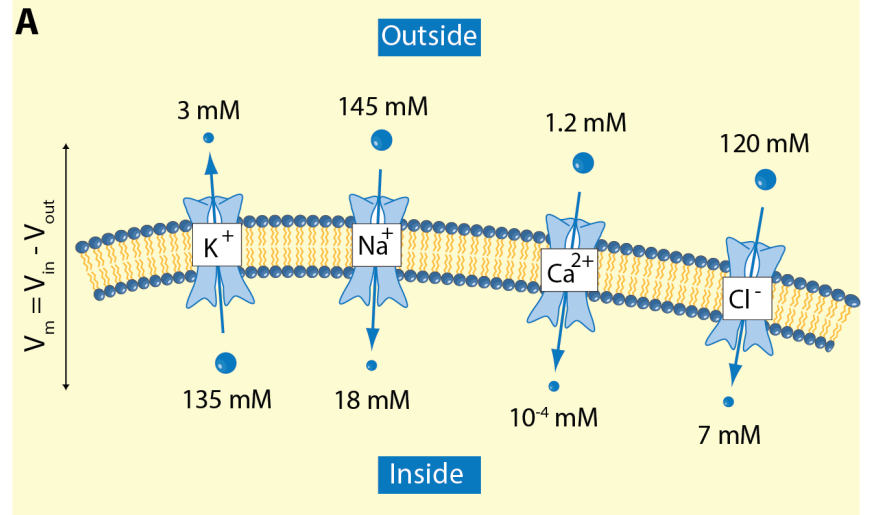

B

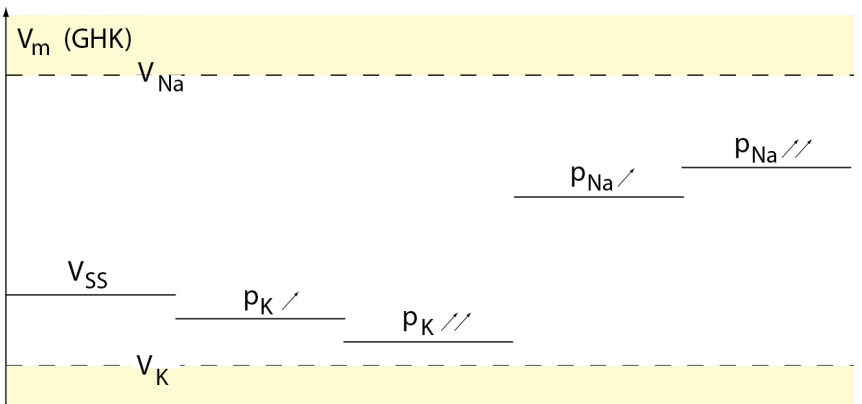

Fig. 2. A flow of ions through the membrane carries changes in neuron membrane potential. A. Ions have a different concentration across the membrane of a neuron. Ion channels (in blue) populate the neuronal membrane and are selectively permeable to one or several ions. The four main ions involved in cell signaling are sodium $\mathrm{Na}^{+}$, potassium $\mathrm{K}^{+}$, calcium $\mathrm{Ca}^{2+}$ and chloride $\mathrm{Cl}^{-}$ions (the concentrations shown in the figure are typical for a mammalian neuron). These concentration gradients are responsible for a voltage gradient across the membrane, giving rise to a membrane potential $V_{m}$. B. Variations of $\mathrm{K}^{+}$and $\mathrm{Na}^{+}$membrane permeabilities affect the membrane potential in opposite ways. Values are computed using the Goldman-Hodgkin-Katz (GHK) equation, considering $\mathrm{K}^{+}$and $\mathrm{Na}^{+}$ions only. Ion concentrations are as in A. An increase in $\mathrm{K}^{+}$ permeability hyperpolarizes the cell $\left(V_{m}\right.$ decreases), whereas an increase in $\mathrm{Na}^{+}$permeability depolarizes the cell ( $V_{m}$ increases). The membrane potential is always contained in the range $V_{K} \leq V m \leq V_{N a}$. Adapted from [7]. 
step $\Delta V$ around a nominal voltage $V$ provides an estimate of the instantaneous conductance $g(V, t)$ through the relationship

$$
\Delta I(t)=g(V, t) \Delta V
$$

In control terms, the voltage-clamp experiment is nothing but the step input response of a one-port electrical circuit, and the conductance is nothing but the local gain of the circuit at a given voltage. Hodgkin and Huxley repeated that input step experiment for the whole range of voltages comprised between $E_{K}$ and $E_{N a}$, thereby obtaining the total ionic conductance of the circuit. They then repeated the same experiment in a sodium free solution, obtaining this time the potassium conductance

$$
\Delta I_{K}(t)=g_{K}(V, t) \Delta V
$$

Finally, they subtracted the potassium conductance from the total conductance to obtain the sodium conductance. The result of their experiments is illustrated in Figure 3 for two values of the input voltage.

The authors identified a conductance model from those step input experiments. The delayed first-order kinetics of the potassium conductance was modeled as

$$
\begin{aligned}
g_{K} & =\bar{g}_{K} n^{4} \\
\dot{n} & =\alpha_{n}(V)(1-n)-\beta_{n}(V) n
\end{aligned}
$$

where $\bar{g}_{K}$ is now a constant parameter termed the maximal conductance, that is, the conductance when the gating variable $n$ reaches its maximum. This variable, normalized in the range $[0,1]$, dynamically modulates the conductance behavior as a function of the voltage. The dynamical equation is often rewritten in the form

$$
\tau_{n}(V) \dot{n}=-n+n_{\infty}(V)
$$

where $\tau_{n}(V)$ and $n_{\infty}(V)$ have the convenient interpretation of a time-constant and a static gain, respectively, each dependent on voltage. To model the sodium conductance, the authors chose the equations

$$
g_{N a}=\bar{g}_{N a} m^{3} h
$$

where the gating variables $m$ and $h$ both obey a first order differential equation of the same type as (8) or (9). The data fit chosen by Hodgkin and Huxley is illustrated in Figure 4.

Analytical expressions from the original paper are omitted here to emphasize that those curves only result from curve fitting. Only the qualitative properties of those matter for the neuronal behavior. The static gain curves are sigmoidal and indicate a monotonic dependence in the voltage. The slope is positive for $m$ and $n$, which are therefore called activation gating variables, whereas it is negative for $h$, which is therefore called a inactivation gating variable. The time constants are also voltage dependent, but their most important feature is that the time-constant of the sodium activation $m$ is five to ten times smaller than the timeconstants of the sodium inactivation $h$ and the potassium activation $n$. The conductance dynamics therefore exhibit
A

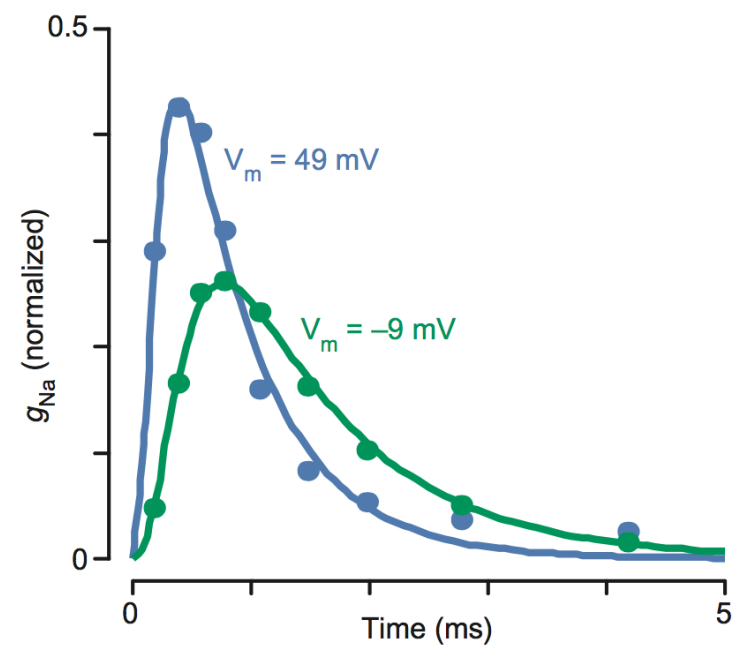

B

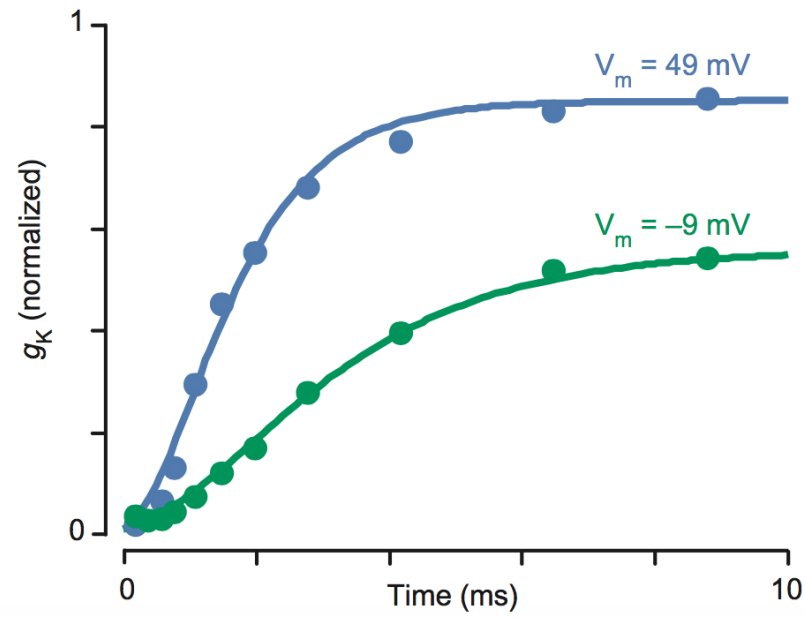

Fig. 3. Experimental voltage-clamp data illustrating the temporal evolution of the sodium (A) and potassium conductances (B) for two values of the membrane potential (green and blue curves, respectivelly) [23]. Reproduced from [4].

two distinct time scales: a fast sodium activation, and a slow sodium inactivation and potassium activation.

Sixty years after Hodgkin and Huxley, the voltage-clamp experiment is still the experiment of choice to model a specific current type. If a pharmacological blocker is known to block the current under investigation, its conductance can be modeled by following the steps of Hodgkin and Huxley experiment. In today's publications, a voltage-gated ionic current typically employs the generic model

$$
g_{i}=\bar{g}_{i} m_{i}^{\gamma} h_{i}^{\delta}
$$

and a first-order kinetics for the activation variable $m_{i}$ and the inactivation variable $h_{i}$.

The reader should bear in mind that few conductances are modeled today with the precision of Hodgkin and Huxley experiment. This limitation owes to the diversity of current types, the diversity of cell types, and the strong variability of experimental results across cells and across labs for a 
A1

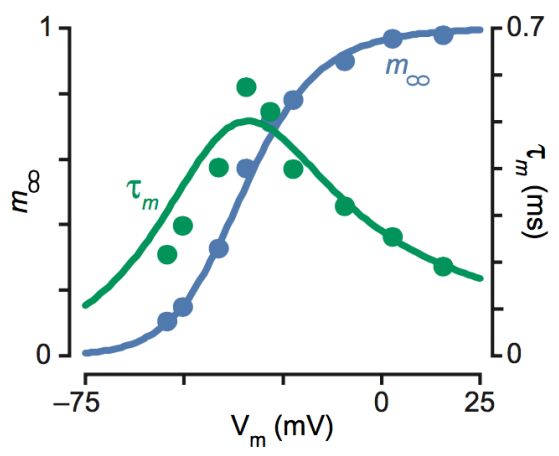

A2

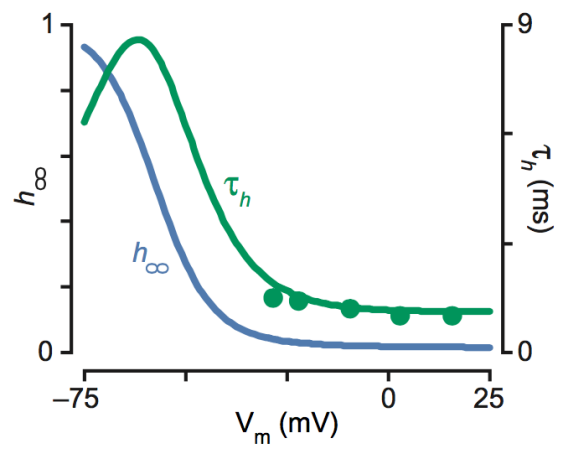

B

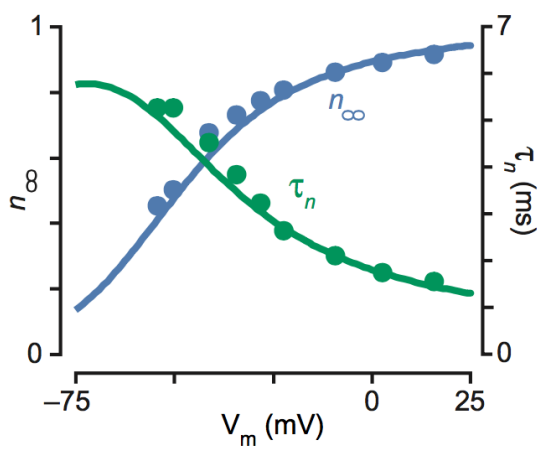

Fig. 4. Voltage-dependence of the time-constants and the static gains of the Hodgkin-Huxley model [23]. The blue curves correspond to the sodium steady-state activation $m_{\infty}\left(V_{m}\right)$, the sodium steady-state inactivation $h_{\infty}\left(V_{m}\right)$ and the potassium steady-state activation $n_{\infty}\left(V_{m}\right)$. The green curves correspond to the sodium activation time-constant $\tau_{m}\left(V_{m}\right)$, the sodium inactivation time-constant $\tau_{h}\left(V_{m}\right)$ and the potassium activation time-constant $\tau_{n}\left(V_{m}\right)$. The dots represent corresponding experimental data. Reproduced from [4].

given current type and given cell type. For this reason, conductance-based models should be regarded as qualitative, and behavioral properties that depend on the quantitative details of the model should be interpreted with care.

2) Conductances can also depend on intracellular concentrations: The ionic currents of Hodgkin and Huxley model only depend on voltage. They are called voltagegated currents. Many current types can also depend on the intracellular concentration of calcium or sodium. For instance, the current $I_{K, S K}$ in [5] is a potassium current that is activated as the intracellular calcium concentration rises. A simple model for the conductance of this current is

$$
g_{K, S K}=\bar{g}_{K, S K} H([C a])
$$

with $H$ a sigmoïdal gating function. The dynamics of the conductance will in this case be determined by the dynamics of the intracellular calcium, which usually obeys a simple mass-balance equation characterized by a time constant and the flow of all calcium ionic currents. The difficulty of measuring intracellular calcium concentrations makes the modeling of calcium-gated conductances even more qualitative than voltage-gated conductances.

It is also common that a conductance depends simultaneously on voltage and calcium. For instance, the conductance of the calcium current $I_{C a, L}$ in [5] combines a voltagedependence modeled with an activation gating variable $m(V)$ and a calcium-dependence modeled with an inactivation gating variable $h([\mathrm{Ca}])$.

3) From conductances to ion channels: Immediately after introducing the equations modeling the potassium conductance, Hodgkin and Huxley discuss their physiological interpretation: these equations may be given a physical basis if we assume that potassium ions can only cross the membrane when four similar particles occupy a certain region of the membrane. $n$ represents the proportion of particles in a certain position (for instance at the inside of the membrane) and $1-n$ represents the proportion that are somewhere else ( for example at the outside of the membrane). $\alpha_{n}$ determines the transfer from outside to inside, while $\beta_{n}$ determines the transfer in the opposite direction [23, p. 507]. This meanfield interpretation of conductance modeling, provided at the time when not much was known about the molecular organization of ion channels, should remind the reader that conductances connect two distinct scales of biological organization: the electrical activity of a cell, that occurs at the microscale, and the biochemical activity of proteins, that occurs at the nanoscale. Ion channels are protein arrangements that control the gating of individual ions through the cellular membrane. Their molecular structure has been the focus of much study in the final decades of the 20th century [22] and has revealed many different types and a great deal of molecular organization. Conductance modeling is an attempt to summarize the collective activity of many ion channels in a lumped model that only depends on few cell variables, such as the membrane potential and some ionic intracellular concentrations or biological signaling molecules.

In short, there are few ionic species that carry current, but there are many channel types. As an example, Figure 5 illustrates the diversity of potassium channels in terms of known families of genes that encode them. Channel expression, the process of biosynthesis and eventual degradation of ion channels in the neuronal membrane, is itself an adaptive process (see Section VI). However, restricting attention to the ion channels present in the membrane at any point in time, it is good to think of ion channels in control-theoretic terms as the "actuators" of the electrical circuit. Each current type corresponds to a population of ion channels characterized by a specific mean-field gain, acting in a specific voltage (or calcium) and frequency range. Besides depending on internal variables, the local gains are also modulated by a realm of molecular processes that involve external quantities such as neurotransmitter concentrations, as well as receptor and channel expression.

\section{A one port circuit regulated by a zoo of biochemical actuators}

A fundamental heritage of Hodgkin-Huxley modeling work is that neuronal behaviors interconnect two behavioral 


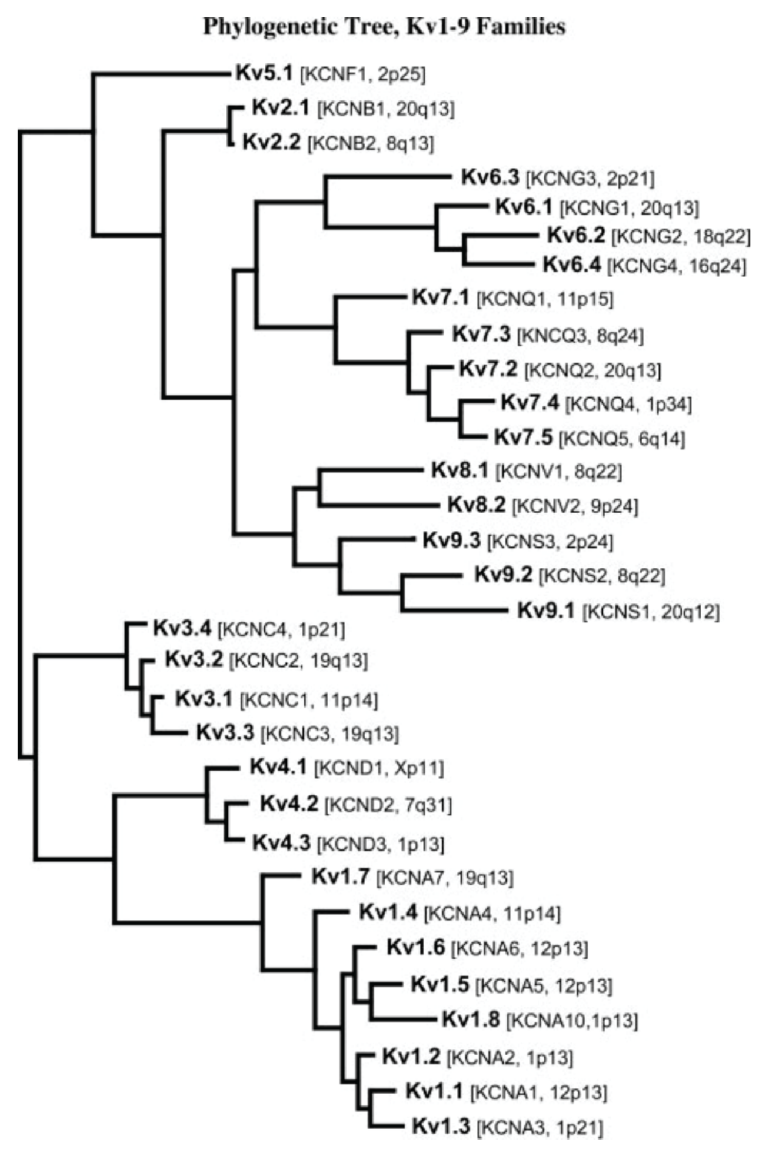

Fig. 5. Ion channel types are very diverse. A phylogenic tree of known voltage-gated potassium channel genes. Reproduced from [19].

scales: an electrical behavior at the cellular (micro-) scale and a biochemical behavior at the molecular (nano-) scale. For a single cell model, the electrical behavior is the elementary behavior of a one-port RC circuit. The capacitive behavior is fast and can be neglected but the resistive behavior is complex, i.e. nonlinear and time-varying. The biochemical behavior must account for the dynamics of thousand of ion channels that, collectively, determine the circuit conductance.

As knowledge accumulates about the molecular processes that regulate ion channel activity, conductance-based models include more and more gating variables, leading to highdimensional nonlinear models of increasing complexity. The separation of the electrical behavior from the biochemical behavior is crucial for the tractability of those models. For a single cell, the electrical behavior specifies a scalar relationship $F(I, V, t)=0$ which, locally in time and range, is entirely characterized by the local description $\Delta I(t)=$ $g(V, t) \Delta V(t)$. In that sense, the instantaneous conductance $g(V, t)$ is a scalar quantity that completely determines the electrical behavior.

In turn, the biochemical behavior has a highly parallel structure. The instantaneous conductance is indeed decomposed as the sum of possibly many independent conductances, each modeling the collective behavior of one type of molecular process. In particular, the (mean-field) input- output behavior from any internal state of the cell (voltage or ionic intracellular concentration) to any gating variable is nonlinear but elementary, that is, entirely characterized by a time-constant and a sigmoidal static relationship.

The very particular interconnection structure of a conductance based-model is key to a tractable system analysis. Each of the possibly many gating variables can be regarded as shaping the one-port circuit conductance in a specific temporal and voltage window. The sensitivity window is determined by the time-constant of the gating variable, and by the voltage range of its static behavior. Parameters, receptors, or neurotransmitters should be regarded as many actuation variables but each having a specific influence on a few gating variables, that is, in a specific window. Because of the highly parallel structure of the biochemical process, the behavior of the one-port circuit in a specific time-voltage window is determined by the few gating variables and related biochemical actuators that have non-negligible sensitivity in that particular window.

In short, the Hodgkin and Huxley heritage is that a neuronal behavior is the behavior of a simple electrical circuit but that its actuation is extraordinary diverse, localized, and adaptive because it is the result of a biochemical behavior at a finer scale. In that sense, conductance-based models are a model of control and regulation across scales.

\section{EXCITABLE BEHAVIORS}

\section{A. Positive and negative feedback}

The resistive part of a $R C$ circuit has the interpretation of a feedback loop around the capacitor, the sign of which is determined by the slope of the resistive curve, i.e. by the sign of the (differential) conductance. In a conventional circuit, positive resistance is synonym of negative feedback and negative resistance is synonym of positive feedback. Due to the sign convention in electrophysiology, a positive conductance in a neuronal model is synonym of negative feedback for an outward current (such as potassium) but of positive feedback for an inward current (such as sodium or calcium).

The experimental results of Hodgkin and Huxley in Figure 3 reveal a fundamental difference between the role of the sodium and potassium in shaping the local (differential) conductance of the circuit. For a small step change from a fixed membrane potential, the local potassium conductance is always positive, that is, the potassium current is always a source of negative feedback. In contrast, the local sodium conductance is initially positive and then becomes negative, which means that the sodium current acts transiently as a source of positive feedback and then as a source of negative feedback.

The conductance model proposed by Hodgkin and Huxley captures this important property: the activation of the sodium current is modeled as a fast monotone behavior that accounts for the source of (fast) positive feedback, whereas its inactivation is modeled as a slower monotone behavior of the opposite sign, which accounts for the source of (slow) negative feedback. The potassium conductance 
is modeled with a single activation variable whose slow monotone behavior accounts for the source of (slow) negative feedback.

The fact that a specific ionic current can be both a source of positive and negative feedback depending on the voltage and time window is important if it is to be understood as a local loop shaper of the circuit feedback gain. This feature is often a source of confusion in the neurodynamics literature. For instance, the sodium current of Hodgkin Huxley is both a source of positive and negative feedback. Its activation makes it a positive feedback in the fast timescale but its inactivation makes it a negative feedback in the slow timescale. In the terminology of [15], the sodium current is fast regenerative but slow restorative. In the literature, this current will often be qualified as an excitatory or depolarizing current, because it is indeed the source of excitability in the neuron, but this terminology is confusing if 'excitatory' or 'depolarizing' is interpreted as a synonym of positive feedback. Figure 6 lists the most common voltage-gated ionic currents found in the literature. It illustrates for instance that the A-potassium current can be a source of positive feedback in the slow time scale, whereas a potassium current would normally be qualified as inhibitory or hyperpolarizing. But a slow inactivation of a hyperpolarizing current makes it a source of positive feedback in the corresponding timescale.

\begin{tabular}{|c|c|c|}
\hline Ionic current & Current flow & Feedback(s) \\
\hline $\begin{array}{l}\text { Transient } \\
\mathrm{Na}^{+} \text {current }\end{array}$ & inward & $\begin{array}{l}\text { Fast regenerative (activation) } \\
\text { Slow restorative (inactivation) }\end{array}$ \\
\hline $\begin{array}{l}\text { Delayed rectifier } \\
\mathrm{K}^{+} \text {current }\end{array}$ & outward & Slow restorative (activation) \\
\hline $\begin{array}{l}\text { L-type } \\
\mathrm{Ca}^{2+} \text { current }\end{array}$ & inward & Slow regenerative (activation) \\
\hline $\begin{array}{l}\text { A-type } \\
\mathrm{K}^{+} \text {current }\end{array}$ & outward & $\begin{array}{l}\text { Fast restorative (activation) } \\
\text { Slow regenerative (inactivation) }\end{array}$ \\
\hline $\begin{array}{l}\text { T-type } \\
\mathrm{Ca}^{2+} \text { current }\end{array}$ & inward & $\begin{array}{l}\text { Slow regenerative (activation) } \\
\text { Ultraslow restorative (inactivation) }\end{array}$ \\
\hline $\mathrm{H}$-current & inward & Ultraslow restorative (activation) \\
\hline $\begin{array}{l}\mathrm{Ca}^{2+} \text {-activated } \\
\mathrm{K}^{+} \text {current }\end{array}$ & outward & Ultraslow restorative (activation) \\
\hline
\end{tabular}

Fig. 6. List of the most common ionic currents shaping the electrophysiological properties of neurons. The left column lists the name of the different ionic currents, the center column provides their inward/outward properties and the right column their regenerative/restorative properties. Note that both inward and outward currents can be either regenerative or restorative in different timescales.

\section{B. Feedback amplifiers: switches, regulators, and resonators}

The development of the feedback amplifier at the turn of the 20th century provides an insightful historical account of how engineers came to appreciate the distinct role of positive and negative feedback in technology. As described

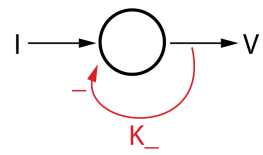

Linear - Regulator (|k| large)

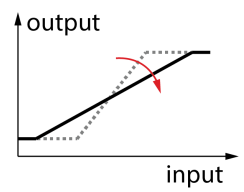

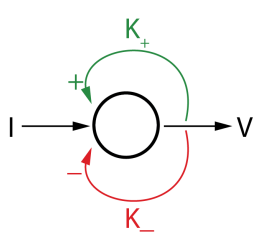

Ultrasensitive- Resonant ( $|\mathrm{k}|$ small)

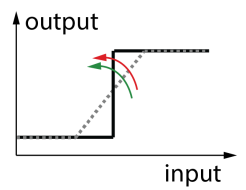

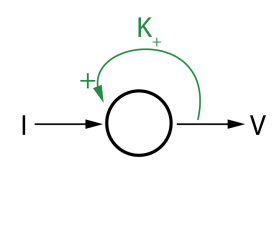

Hysteretic - Switch (|k| large)

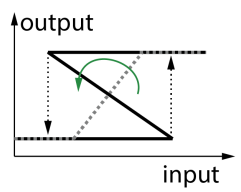

Fig. 7. Regulating the balance between positive and negative feedback can switch a system between linear, ultrasensitive and hysteretic states. Top, sketches of the systems composed of a negative feedback (left), a positive feedback (right), or both (center). Bottom, input/output relationships in the three cases. The dashed grey lines show the open-loop relationships, and the full black lines the closed-loop relationships.

in [41], only positive feedback amplifiers (also called regenerative amplifiers) played an important role initially, until the dramatic invention of the negative feedback amplifier in 1927 made the latter the pillar of control theory and turned the former into history. Biology suggests that both are equally important in the organization of natural behaviors. The complementary role of negative and positive feedback shaped the development of cybernetics [1], but the invention of the computer made digital technology (akin to positive feedback) more and more separated from analog technology (akin to negative feedback).

To appreciate the difference between positive and negative feedback, the static theory of the feedback amplifier is a useful starting point. The static behavior of the openloop amplifier is a finite-range sigmoidal behavior, well approximated by a linear behavior between two saturation levels. For the convenience of the discussion, assume that the amplifier has saturation levels 0 and 1 and a linear gain $k$ over a range $1 / k$ (Figure 7 ).

A negative feedback amplifier subtracts to the input the value of the output multiplied by the feedback gain $K>0$. The closed-loop behavior is a new amplifier with the same saturation levels but with a new (closed-loop) gain equal to $\frac{k}{1+K k}$ over a range $\frac{1+K k}{k}$. This means that negative feedback decreases the open-loop gain and spreads the linear range of the behavior. Negative feedback linearizes, that is, it spreads the linear sensitivity of the behavior over a broader range (Figure 7, right). If the open-loop gain is high, the closedloop gain is $O\left(\frac{1}{K}\right)$ over a range $O(K)$. Over its linear range, the feedback amplifier is a regulator. The behavior is exogenous: the output voltage is proportional to the input voltage, and the gain of the amplifier is insensitive to its internal behavior. Negative feedback is the essence of control theory.

A positive feedback amplifier adds to the input the value of the output multiplied by the feedback gain $K$. For a value $K>\frac{1}{k}$, the closed-loop behavior becomes multi-valued 
over a range $\frac{1+K k}{k}$. The two saturation levels coexist in that range, together with the linear behavior. Because of its negative slope, the linear behavior is unstable, so that it is not observed in the presence of dynamics or perturbation. Hence, positive feedback turns the amplifier into an hysteretic switch (Figure 7, right). For a large open-loop gain, the range of the hysteresis is proportional to the feedback gain. Over its hysteretic range, the amplifier is a switch, that is, a two-state automaton. The behavior is endogenous: the output voltage is 0 or 1 , regardless of external input voltage variations.

When an amplifier combines a positive feedback loop of gain $K_{+}$with a negative feedback loop of gain $K_{-}$, the closed-loop behavior is either a regulator or a switch. Those two behaviors are separated by a singular behavior for the particular value $K_{+}-K_{-}=\frac{1}{k}$. Near this singular value, the behavior is resonant, characterized by an ultrasensitive switch or an ultra-sensitive amplifier over a very narrow range (Figure 7, center). This ultra sensitive behavior deserves more attention in control theory than it has received in the past. It seems to play a fundamental role in the organization of natural behaviors [39].

\section{Excitability as robust ultrasensitivity}

The ultra-sensitive behavior obtained when positive feedback suitably balances negative feedback may appear as a fragile construction. But it can be turned into a robust mechanism by adding a dynamical component to the static picture. If the negative feedback dominates in static conditions, but the time constant of the positive feedback is much smaller than the time constant of the negative feedback, then the dynamical behavior of the closed-loop amplifier is de facto dominated by positive feedback at high-frequency and is dominated by negative feedback at low frequency. By continuity, the behavior must be resonant, or ultra sensitive, in a specific frequency range and a specific input-range. This is the essence of excitable behaviors.

The state-space model of the mixed feedback amplifier in Figure 8 is

$$
\begin{aligned}
\tau_{f} \dot{x}_{f} & =-x_{f}+K_{+} V \\
\tau_{s} \dot{x}_{s} & =-x_{s}+K_{-} V \\
V & =S\left(u+x_{f}-x_{s}\right)
\end{aligned}
$$

If we assume enough separation between the fast timescale $\tau_{f}$ and the slow timescale $\tau_{s}$, the mixed feedback amplifier exhibits a two timescale behavior: the fast behavior, which assumes that $x_{s}$ is a fixed parameter, is the behavior of a positive feedback amplifier: the voltage is discrete over a range $O\left(K_{+}\right)$and insensitive to variations of the input $u-x_{s}$.

For an ideal amplifier, the static behavior of the mixed feedback amplifier is the behavior of a negative feedback amplifier provided that $K_{-}>K_{+}$. The amplifier has the exogenous behavior $V=\left(K_{-}-K_{+}\right)^{-1} u$ over its linear range $O\left(K_{-}-K_{+}\right)$. But this behavior is a static approximation of the dynamical behavior. The dynamical behavior is a mix of a switch and a regulator. For a fixed input $\bar{u}$, the steady-state behavior is either a stable equilibrium at

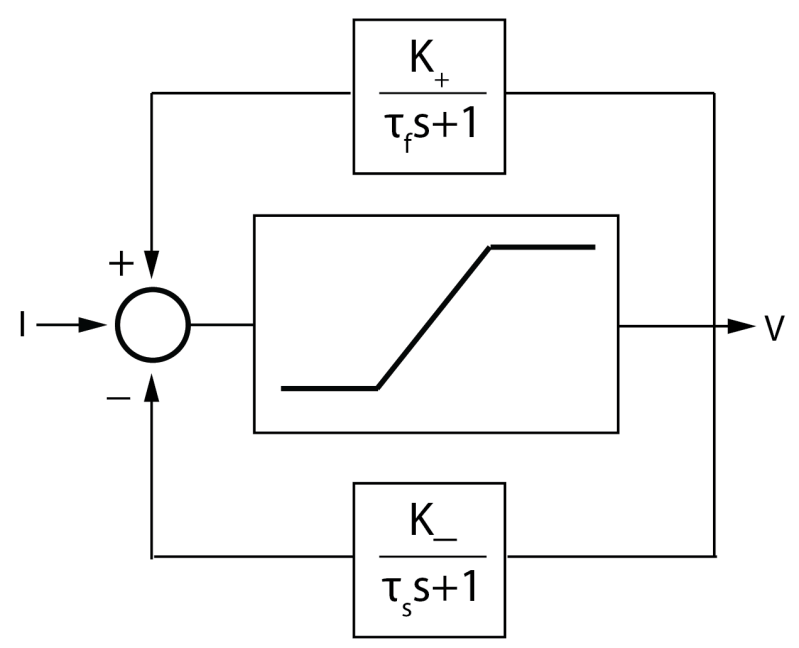

Fig. 8. Block diagram representation of the mixed feedback amplifier. The central block is a sigmoid function. Two feedbacks control the input/output properties of the mixed feedback amplified: a fast, positive feedback (gain: $K_{+}$, time-constant: $\tau_{f}$ ) and a slow, negative feedback (gain: $K_{-}$, time-constant: $\tau_{s}$ )

$x_{1}=0$ or $x_{1}=1$, or a stable oscillation of amplitude 1 and frequency determined by the filter time constants. The excitable behavior is determined by the regime where the resting state $x_{1}=0$ is stable but where (small) transient inputs can cause (large) transient excursions to the excited state $x_{1}=1$. In the excitable regime, the behavior is ultra sensitive, that is, excursions to the excited state can be triggered by arbitrarily small perturbations in the right amplitude and frequency range.

\section{Neuronal excitability and its organizing singularity}

The last part of Hodgkin and Huxley 1952 paper is a simulation (a formidable task at the time) of the four nonlinear differential equations to reproduce the action potential, i.e. the large and brief pulse response to a pulse current input above threshold. See Figure 9. The circuit mechanism of the action potential is elementary: the fast activation of the sodium current acts as a switch (or positive feedback or autocatalytic process). But this switch is only transient, both because of the slow inactivation of the sodium current and the slow activation of the potassium current. Potassium current activation is responsible for the hyperpolarized transient following the spike (also called refractory period).

The excitable behavior of Hodgkin-Huxley circuit is closely related to the behavior of the mixed feedback amplifier. The circuit equation

$$
0=I_{N a}(V, h, m)+I_{K}(V, n)+I_{l}(V)+I
$$

relating the applied current $I$ to the voltage $V$ is analog to the amplifier equation (14) relating $u$ to $V$. Its local behavior has the interpretation of a feedback gain modulated by a twotimescale system. The sodium activation $m$ is the analog of the variable $x_{f}$ : it provides a source of positive feedback in the fast timescale. The sodium inactivation $h$ and the potassium activation $n$ are the analog of the variable $x_{s}$ : they provide a source of negative feedback in the slow timescale. 


\section{Action potential generation}

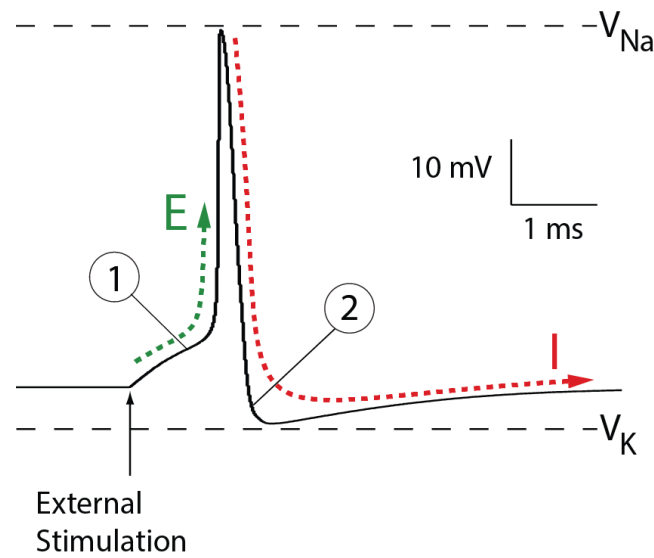

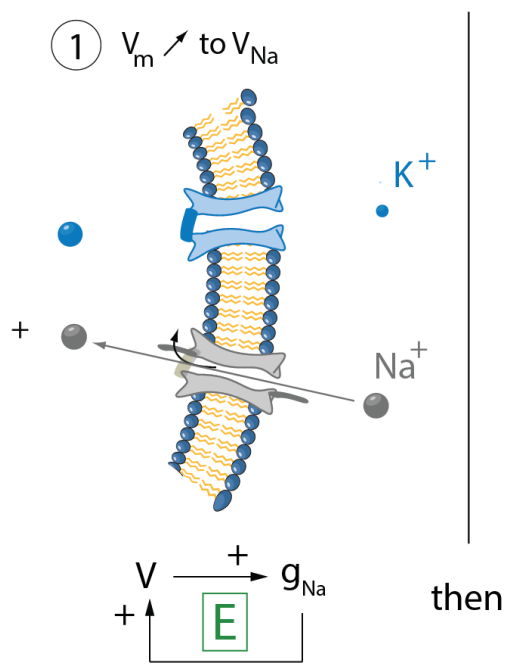

(2) $v_{m} \backslash$ to $V_{k}$
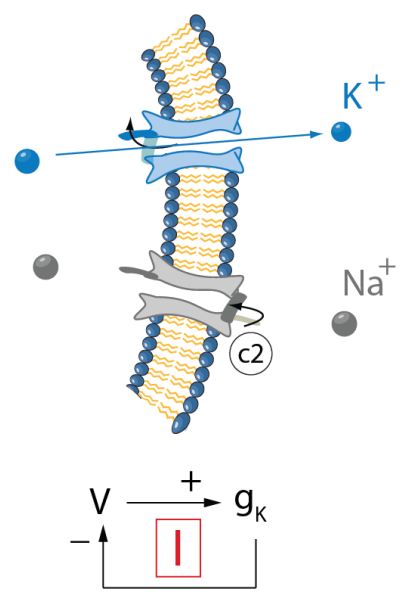

Fig. 9. HH model is an E-I motif. Left, membrane potential variations over time during an action potential generation. Green and red dashed arrows depicts regenerative and restorative events, respectively. Right, sketch of ion channels embedded in neuron plasma membrane showing ion channel voltage-gating (top) and corresponding feedback loops (bottom). Adapted from [7].

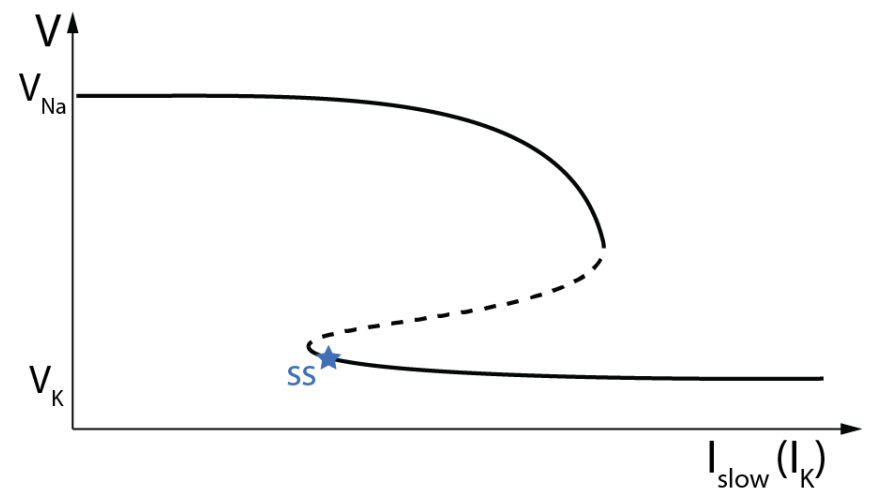

$\operatorname{Neg} F B\left(I_{k}\right)$

Fig. 10. Hysteretic relationship between membrane potential and membrane current in the fast subsystem of the Hodgkin-Huxley model. The figure shows the steady-states of the circuit equation $(0=$ $\left.I_{N a}(V, h, m)+I_{K}(V, n)+I_{l}(V)+I\right)$ where the fast variable $m$ has converged to its steady-state $m_{\infty}(V)$ and slow variables $h$ and $n$ are treated as parameters. Full black lines represent stable fixed points dashed black lines represent unstable fixed points (saddle points in this case) and the blue star depicts model steady-state (SS). Neg FB = negative feedback.

Figure 10 illustrates the hysteretic relationship between the slow current $I_{\text {slow }}$ and $V$ in Hodgkin and Huxley model when the fast variable $m$ has converged to its steady-state $m_{\infty}(V)$ and the slow variables are treated as parameters. It is qualitatively similar to the hysteretic relationship between $u$ and $V$ in the mixed feedback amplifier when the fast variable $x_{f}$ has converged to its steady-state $K_{+} V$ and the slow variable $x_{s}$ is treated as a parameter.

The essence of an excitable behavior is thus a modulation of a scalar behavior from hysteretic on the fast timescale, when the positive feedback is on, to monotone on the slow timescale, when the positive feedback is off. The normal form

of this transition is given by

$$
F(x, \beta, \lambda)=0 \equiv 0=\beta x-x^{3}+\lambda
$$

which, in the language of singularity theory [37] is the universal unfolding of the hysteresis singularity $x^{3}+\lambda$. The parameter $\lambda$ is a control parameter that determines the system state according to the characteristic $F(x, \beta, \lambda)=0$. The parameter $\beta$ is an unfolding parameter: it organizes the possible characteristics of the model into two distinct families: those that correspond to the hysteretic curve $(\beta>$ $0)$, and those that correspond to the monotone behavior $(\beta<0)$.

The celebrated Fitzugh Nagumo model [12]

$$
\begin{aligned}
C \dot{V} & =\beta V-\frac{V^{3}}{3}+n+I \\
\dot{n} & =\epsilon(-n+V)
\end{aligned}
$$

is a normal form of excitable behaviors: it is a two-timescale behavior whose slow dynamics modulate the bifurcation parameter of a fast behavior organized by a hysteresis singularity. It captures the qualitative behavior of HodgkinHuxley model and of the mixed feedback amplifier. It is also equivalent to the negative resistance oscillator of Van der Pol (with a constant current source) and gives rise to the classical phase portrait of excitability. In the fast time scale, that is, for a nearly constant $n$, the circuit is a bistable switch, a consequence of the locally positive feedback. In the slow timescale, the dynamics of the recovery variable $n$ provides adaptation, a consequence of the global negative feedback.

\section{E. Excitability: mixing the best of two distinct worlds}

As a basic element of biological behaviors, the excitable behavior, defined as a nonlinear resistive circuit that is locally dominated by positive feedback and globally dominated by negative feedback, shares features of both the positive and the negative feedback amplifiers. Like the positive feedback 
amplifier, it exhibits the binary nature of a one state automaton, with a well identified resting state (0) and excited state (1). But the negative feedback loop regulates the switch between the high and low state, that is, the transient from the resting state to the excited state (spike generation), and the transient from the excited state to the resting state (after-spike hyperpolarization). Like the negative feedback amplifier, the excitable behavior exhibits the analog nature of a continuously regulated modulation between the two discrete states.

The hybrid nature of the excitable behavior calls for a modeling framework that is neither the discrete framework of automata nor the continuous framework of differential equations, but a mix of both, that is a hybrid model [16]. This should not suggest however, that the excitable behavior is even less tractable than an automaton or a set of differential equations. Instead, one should acknowledge the specific property that an excitable behavior is organized by a singularity, meaning that its analysis is amenable to a local analysis in state, parameter, and input spaces.

\section{F. Two-level excitability: bursting, pacemaking, tonic firing}

The localization of excitable behaviors is key to the tractability of their analysis because localized behaviors qualitatively obey a superposition principle. We will illustrate this property by extending the above analysis of Hodgkin and Huxley model to the analysis of a circuit that contain two rather than one local sources of positive feedback surrounded by global sources of negative feedback. The motif of this circuit is illustrated in Figure 11A: it consists of a first source of positive feedback localized in a high voltage range and high frequency range, like in Hodgkin and Huxley model, augmented with a second source of positive feedback localized in a lower voltage range and a lower frequency range.

This motif can be realized physiologically with two additional ionic currents with respect to Hodgkin-Huxley model. Such currents abound in the literature and are in fact a hallmark of neurons exhibiting bursting, a specific firing pattern that consists in the alternation of hyperpolarized resting periods and periods of high frequency spiking. The T-type calcium current $I_{C a, T}$ in Fig. $1 \mathrm{~B}$ is an example of physiological current that qualifies as a second source of excitability, distinct from the sodium activation: this current is normally modeled in the same way as the sodium current of Hodgkin-Huxley model, except that its activation range is significantly lower (in the literature, it is referred to as a low-treshold current) and its activation time-constant is about five to ten time slower. The L-type calcium current in the dopaminergic neuron of Figure $1 \mathrm{~B}$ also qualifies for a similar second source of excitability. Those local sources of positive feedback are always counteracted by global sources of negative feedback, coming from their inactivation or from distinct currents. For instance, the activation of the current $I_{K, C a}$, a calcium-activated potassium current, is a negative feedback that specifically counteracts the positive feedback of calcium activation, in a slower timescale than the activation of the potassium current of Hodgkin-Huxley model.

How complex can be a circuit behavior that combines two local sources of excitability? Answering this question from a bifurcation analysis of the conductance based-model is not tractable. The number of dimensions and parameters rapidly rises and makes the analysis complex and fragile. In contrast, the discrete nature of excitable behaviors suggests that the new underlying automaton has two discrete states rather than one. The two-state automaton has four distinct states: a passive state $(00)$, with both sources of positive feedback turned off, a high excited state $(10)$, with the fast positive feedback turned on and the slow positive feedback turned off, a low excited state (01) with the fast positive feedback turned off and the slow positive feedback turned on, and a mixed excited state (11), with both sources of positive feedback turned on. Those four states indeed correlate well with the four endogenous states of most neuronal models: the passive/not excitable state of the early phases of cell development, the fast sodium spike of Hodgkin-Huxley model, the slow calcium spike of models deprived from sodium, and the burst, which superposes fast and slow spikes. The experimental recording in Figure 11B illustrates two out of the four endogenous states of a bursting neuron: in the initial phase of the experiment, the neuron exhibits the fast spike of the high E-I motif, which is the behavior of Hodgkin-Huxley model. Following a step of hyperpolarizing input, the low source of excitability is activated and the neuron exhibits the mixed fast-slow spike of the high+low E-I motif. Further hyperpolarization of the neuron leads to a mixed excitable resting state, with both sources of excitability latently switched on.

The richness of neuronal behaviors observed in singlecell electrophysiology does not seem to require more discrete states. Instead, it is the continuous regulation of the two local sources of positive feedback by many distinct sources of negative feedback that provides a continuous interpolation between the four discrete states of the automaton. Figure 12 illustrates the experimental transition from slow firing to burst firing in different types of neurons. Different types of ionic currents are at play in different types of neurons, resulting in quantitative differences in the different switches, but the qualitative switch is the same in the four situations: the burst oscillation results from activation of a low source of positive feedback.

How tractable is the analysis of such a diversity of nonlinear behaviors? As for Hodgkin-Huxley model, it is the localization of the excitable behavior that makes the analysis of the two state automaton tractable. For the two-level E-I motif localization is around the balance of the slow positive and slow negative feedback in the algebraic relationship:

$$
\begin{aligned}
0= & I_{N a}(V, h, m)+I_{K}(V, n)+I_{C a}\left(V, m_{C a}\right) \\
& +I_{K, C a}(V,[C a])+I_{l}(V)+I
\end{aligned}
$$

Figure 13 shows the dependence of the fast steady-state $(V, m)=\left(V, m_{\infty}(V)\right)$ on the slow current $I_{\text {slow }}$, as determined by (17). Variations of the slow current are generated 
A

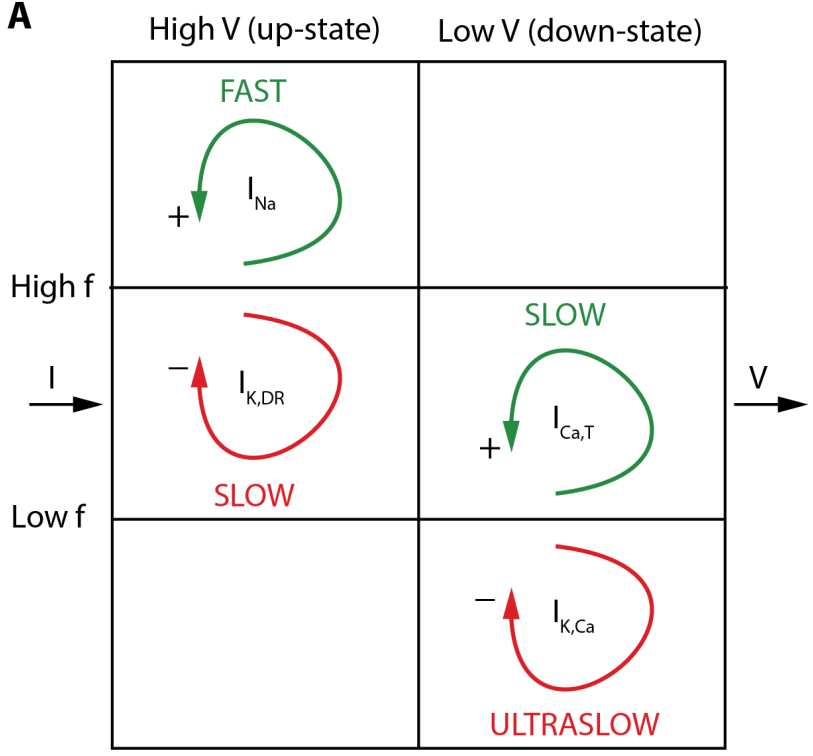

B Hyperpolarization-induced bursting

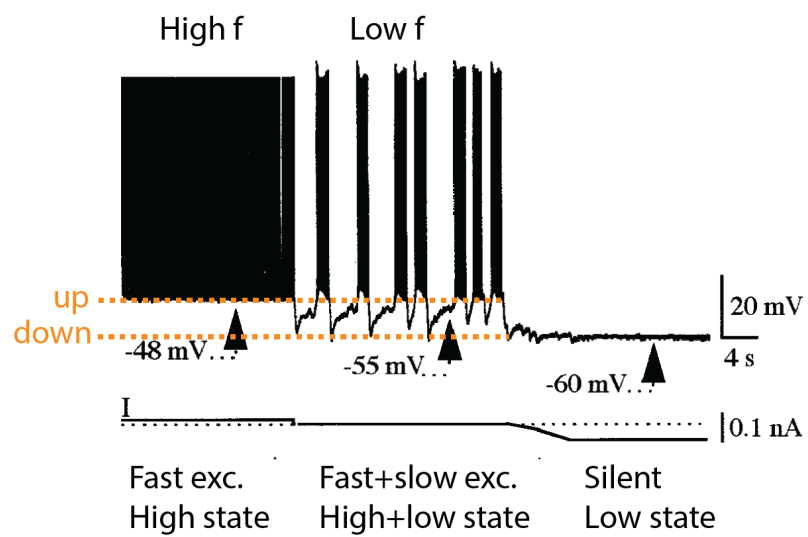

Fig. 11. An experimental recording exhibiting two of the four-state automaton of a bursting neuron. (A) Scheme of the four-state automaton showing the different feedbacks and their localization in frequency (from top to bottom) and in range (from left to right). Green arrows represent positive feedbacks and red arrows represent negative feedbacks. Ionic currents responsible for the described feedbacks are noted inside the respective arrows. (B) Experimental recording of a subthalamic nucleus neuron [2]. The neuron switches from tonic spiking to bursting as its membrane is hyperpolarized via the application of an external current. Further hyperpolarization leads to the suppression of the oscillatory activity.

by the joint variation of the slow variables $h, n, m_{C a}$. The qualitative shape of this curve is a mirrored hysteresis, where the mirror is placed at the balance between slow positive and slow negative feedback, that is, where the net slow feedback gain vanishes.

The static behavior of the extended model is organized by a novel singularity, the winged cusp $x^{3}+\lambda^{2}$, that captures the most singular behavior that can occur upon parameter modulation. This singular behavior is obtained for vanishing fast positive feedback and at the balance between slow positive and slow negative feedback. The normal form of
A Reticular cells of the thalamus

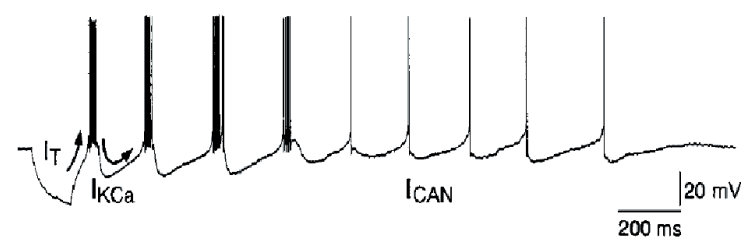

B Midbrain dopaminergic neurons

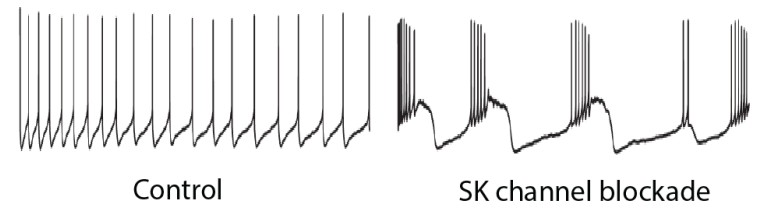

C Relay cells of the thalamus

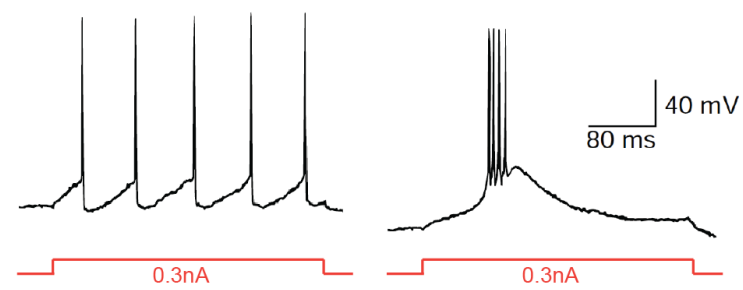

Subthalamic nucleus neurons

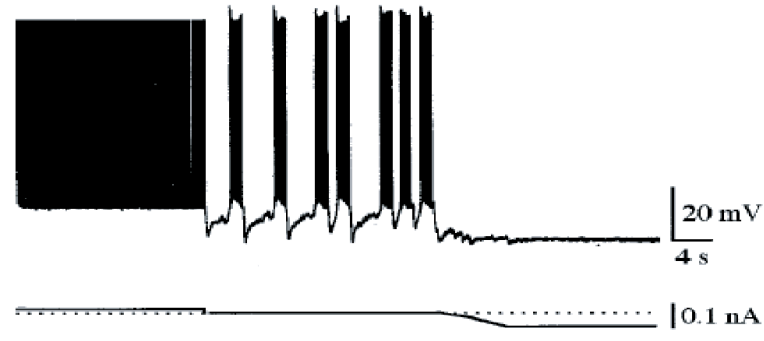

Fig. 12. Routes to bursting in different neuron types. (A) Experimental recording of a thalamic reticular cell [30] exhibiting a smooth switch from bursting to tonic firing in response to transient hyperpolarization. (B) Experimental recordings of a dopaminergic neuron of the substantia nigra pars compacta [25] exhibiting a switch from tonic firing (left) to bursting (right) after the blockade of small conductance calcium-activated potassium (SK) channels. (C) Experimental recordings of a relay cells of the thalamus [40] exhibiting two different behaviors depending on the cell resting potential. A depolarized resting potential leads to tonic spiking (left) whereas a hyperpolarized resting potential leads to bursting (right). (D) Experimental recording of a subthalamic nucleus neuron [2] exhibiting a switch from tonic firing to bursting to silence as described in Fig. 11

its universal unfolding is

$$
F(x, \alpha, \beta, \gamma, \lambda)=0 \equiv 0=\beta x-x^{3}+\lambda^{2}+\alpha+\gamma \lambda x
$$

Mimicking the two-time scale analysis of excitable behaviors, the model

$$
\begin{aligned}
C \dot{V} & =k V-\frac{V^{3}}{3}+\left(n+n_{0}\right)^{2}+I-z \\
\dot{n} & =\epsilon_{n}(V)\left(-n+n_{\infty}\left(V-V_{0}\right)\right) \\
\dot{z} & =\epsilon_{z}(V)\left(-z+z_{\infty}\left(V-V_{1}\right)\right)
\end{aligned}
$$




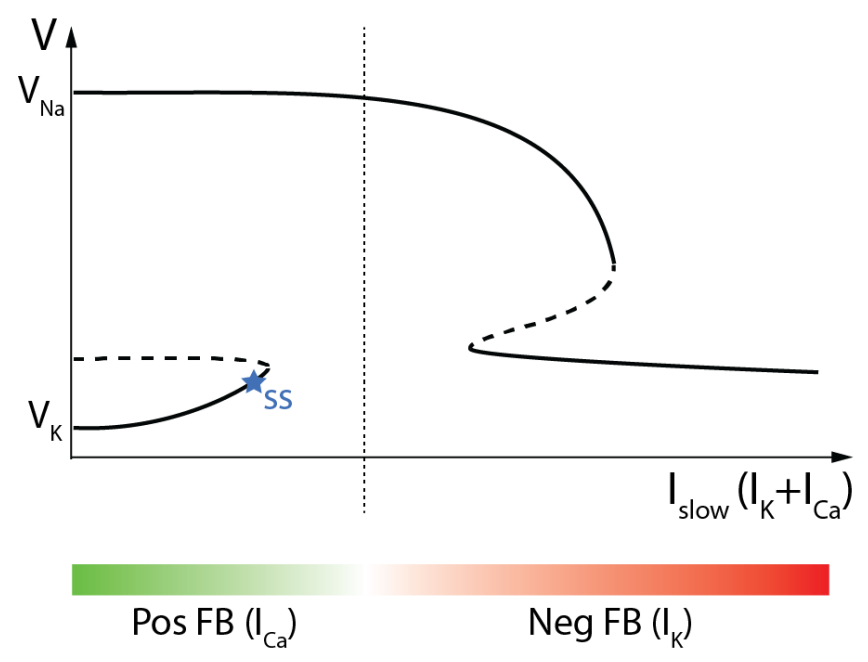

Fig. 13. Mirrored-hysteretic relationship between membrane potential and membrane current in the fast subsystem of the Hodgkin-Huxley model with calcium channels. The figure shows the steady-states of the circuit equation $\left(0=I_{N a}(V, h, m)+I_{K}(V, n)+I_{C a}\left(V, m_{C a}\right)+\right.$ $\left.I_{K, C a}(V,[C a])+I_{l}(V)+I\right)$ where the fast variable $m$ has converged to its steady-state $m_{\infty}(V)$ and slow variables $h$ and $n$ are treated as parameters. Full black lines represent stable fixed points, dashed black lines represent unstable fixed points (saddle points in this case) and the blue star depicts model steady-state (SS). Pos FB = positive feedback and Neg FB = negative feedback.

introduced in [14] captures all three time scale behaviors organized by the cusp, and in particular, the three-time scale behavior of any nonlinear resistive model that combines two distinct sources of positive feedback in two distinct timescales.

As detailed in [14], to which the interested reader is referred for further details, the mathematical model is remarkably consistent with the experimentally observed endogenous behavior of neurons. In particular, there is a oneto-one correspondence between the unfolding parameters of the mathematical model, which capture the continuous modulation between a discrete family of distinct behaviors that can be obtained by an arbitrary variation of the parameters of the scalar relationship (17), and the physiology of the ionic currents that have been identified as key modulators of neuronal excitability.

This illustration from neurophysiology is an encouraging indication that the analysis of excitable behaviors is tractable in nonlinear circuits that combine localized sources of positive and negative feedback. The few local sources of positive feedback determine the dimension of a discrete state automaton of restricted complexity, whereas the possibly many and global sources of negative feedback provide a continuous modulation between those few discrete states.

\section{SENSITIVITY ANALYSIS OF NEURONAL BEHAVIORS}

\section{A. Experimental puzzles for control theorists: an anecdote}

Because the neuronal excitability of a neuron is regulated by possibly many different types of ion channels, an important experimental question for the electrophysiologist is to determine the sensitivity of a neuronal behavior to a change in conductances: which ion channel type is most critical to a particular behavior? Answering such questions may for instance help the pharmacologist to design drugs that will specifically alter a particular ion channel activity in order to restore the physiological function of a neuronal behavior. Experimentally, the sensitivity analysis question is normally addressed through a knock-out experiment: a specific blocker is designed to block a particular channel type, and the neuronal behavior is compared in the presence and in the absence of the blocker.

Our first experimental collaboration was motivated by such a question: we seeked to help our colleague V. Seutin to assess the respective role of L-type calcium channels and sodium channels in the pacemaking activity of midbrain dopaminergic neurons. In normal in vitro conditions, those neurons fire very regularly and endogenously at a low frequency of about $1 \mathrm{~Hz}$. Various labs had tested the outcome of blocking either combination of sodium and/or L-type calcium channels, two distinct sources of positive feedback, with outcomes that often led to conflicting conclusions. For instance, the authors of [20] observed no significant alteration of the rhythm under blockade of L-type calcium channels, concluding that those channels are not involved in the pacemaker activity, whereas the authors of [36] observed that the rhythm was completely disrupted in similar conditions, concluding to the essential role of L-type calcium channels in pacemaking. Such conflicting interpretations of experiments are not rare in electrophysiology.

Can modeling assist experimentation in resolving such questions? The conventional computational way to assist experimentalists in the sensitivity question is to mimic the knock-out experiment in silico: the experimental protocol of blocking the activity of a channel type translates into the computational protocol of reducing the maximal conductance parameter of the corresponding ionic current. Matching the experimental observation with a computational model requires a sufficiently realistic model of the studied neuron. In our study, we were recommended to use a state-of-the art model of the dopaminergic neuron, which contained 130 nonlinear differential equations and more than 500 parameters [5], [8]. In order to shed light on the experimental controversy, we first (empirically) reduced the model to six differential equations and about 20 parameters [10]. Then we used bifurcation analysis to study the transition from pacemaking (slow oscillation) to rest (stable equilibrium) and produced the conceptual diagram in Figure 14. The diagram suggests that the transition from rest (white) to pacemaking (blue) is neatly defined by a (almost linear) combination of the sodium conductance and the L-type calcium conductance. This means that the two currents cooperate to achieve the required neuronal excitability of pacemaking (both currents contribute a positive feedback activation). This cooperation provides a straightforward hypothesis to explain the fragility of the knock-out experiment: small deviations of the nominal point in the parametric plane $\left(\bar{g}_{\mathrm{Ca}, L}, \bar{g}_{\mathrm{Na}}\right)$ are sufficient to generate the four possible outcomes of blocking either of the two channels, suggesting that the intrinsic variability of 
ionic conductances between different neurons would be sufficient to observe different outcomes of a same experimental protocol.

The predictions of our reduced model were easily reproduced in the full computational model, only varying the two considered maximal conductance parameters and keeping all remaining parameters unchanged. As an experimental validation of this hypothesis, we recorded the effect of blocking either channel in a same experimental protocol applied to eleven different dopaminergic neurons. In spite of identical experimental conditions, we observed three of the four possible different outcomes in the population of eleven neurons, suggesting that the degree of cooperation between sodium and L-type calcium considerably varies from one neuron to the other (see [10] for details).

\section{B. Experimental, computational, and mathematical sensitiv- ity analysis}

The anecdote above is for one particular type of ion channel and one particular type of neuron but the lessons of the anecdote have far more generality, most likely beyond the particular field of experimental neurophysiology.

At the experimental level, the lesson of the anecdote is that the knock-out experiment is potentially a fragile experiment in any context where the behavior results from the cooperation of distinct but redundant mechanisms. As explained above, a pacemaking behavior only requires one source of positive feedback and one slower source of negative feedback. But in neurons, a particular balance between these positive and negative feedbacks can be achieved in many ways and regulated by the activation of many different channel types. The cooperation between the sodium and Ltype calcium channels described in our anecdote is one such example. It illustrates the potential difficulty of assessing the role of one particular channel type through one particular knock-out experiment. Both channels contribute to the positive feedback that is necessary for the pacemaking behavior. As a consequence, an identical pacemaking behavior can be achieved with vastly different expressions of a particular ion channel type, and, conversely, identical expression of a particular channel type can lead to vastly different behaviors. This fact is shared by all types of neurons, because all neurons are regulated by many different channel types. And the conclusion is probably far more general, since both the excitable behavior and the redundancy of regulation mechanisms are pervasive across biology.

At the computational level, the lesson of the anecdote is that a computational model is not necessarily of great help to resolve the fragility of the experimental protocol. The physiological realism that is needed to assess the role of a particular channel type in a particular neuron leads to high-dimensional models and high-dimensional parameter spaces. Even in the unlikely situation where a detailed model will perfectly account for the experimental conditions, replacing the experimental sensitivity analysis by a purely computational sensitivity analysis is largely intractable. A computational study like the one reported in [35] illustrates what it takes to explore multiple conductance and connectivity parameters in a particular neuronal model using brute force. But even regardless of the computational effort and of the physiological realism of the conductance-based model, the anecdote above suggests the fragility of the computational outcome. Tiny variations of the "nominal" point in the parameter space may drastically affect the outcome of the computational "global" sensitivity analysis.

The alternative to experimental and computational sensitivity analysis is to resort to mathematical analysis of the neuronal model. Our anecdote could appear as a successful story in that regard, since the proposed explanation came from bifurcation analysis of a reduced model. But the true lesson of the anecdote is that our analysis was completely ad hoc. It took the best of a $\mathrm{PhD}$ project [7] to get the detailed computational model to work, then reduce it empirically to a tractable dimension, then perform a bifurcation analysis of the reduced model, then validate this bifurcation analysis on the full model, then propose an experimental protocol to support the analysis, and finally to perform the experiments. This project could be started only because both a reliable detailed conductance based-model and a lot of experimental data existed for the dopaminergic neuron. Those elements would be lacking for most neurons and will continue to lack for a long time for most biological behaviors.

\section{Can robustness coexist with modulation?}

The discussion in the previous section suggests that the parametric sensitivity analysis of neuronal models is inherently fragile: small variations in the space of physiological parameters may drastically affect the behavioral outcome of large parameter variations. In other words, global sensitivity analysis around a nominal point in parameter space is potentially fragile to small changes in the choice of the nominal point. Such a property by no means implies that the behavior itself is fragile. On the contrary, it is a signature of over-actuated behaviors, that is, behaviors determined by few degrees of freedom but regulated by many cooperating processes. Such behaviors can be at the same time robust to large parameter changes and sensitive to tiny parameter changes: robust because the same behavior can be achieved with vastly different combinations of parameters (provided they determine the few degrees of freedom in the same manner); sensitive because in a given parameter configuration, a tiny change in one parameter is sufficient to drastically affect the response of the system to a given input.

Disentangling the robustness and modulation capabilities of a behavior is a current challenge in experimental neuroscience and, more generally, in biology. Qualitative models aim at capturing the few degrees of freedom that are necessary to account for a given behavior. For instance, the excitable behavior only requires one positive feedback loop and one slower negative feedback loop. In contrast, quantitative models aim at capturing the diversity of regulatory processes and to link them to physiological parameters. But disentangling the robustness and modulation properties of the behavior requires a mapping from physiological parameters 


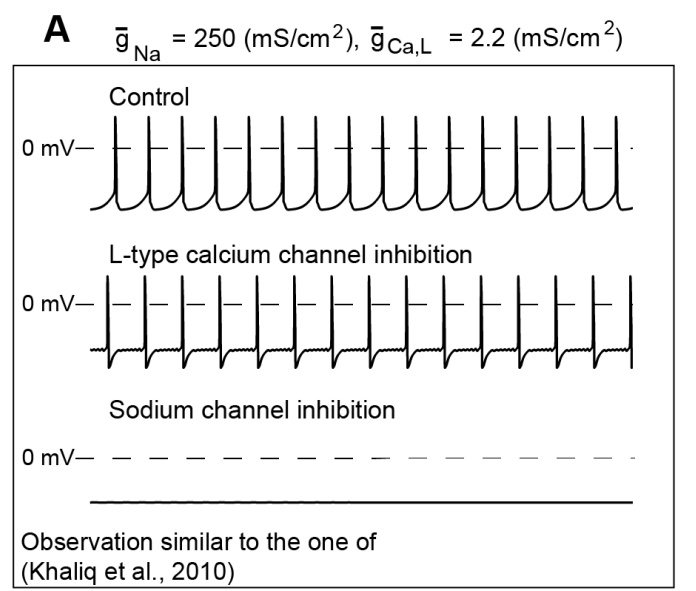

(Khaliq et al., 2010)
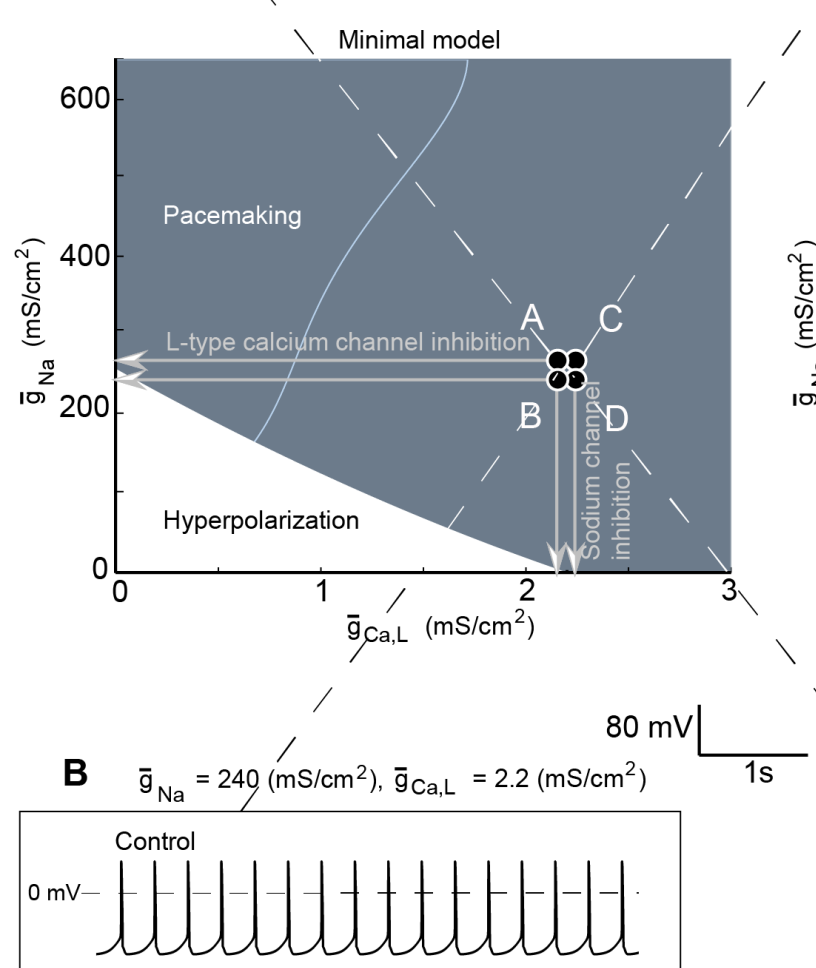

L-type calcium channel inhibition

$0 \mathrm{mV}-----------$

Sodium channel inhibition

$0 \mathrm{mV}---------\cdots$
C $\quad \bar{g}_{\mathrm{Na}}=250\left(\mathrm{mS} / \mathrm{cm}^{2}\right), \bar{g}_{\mathrm{Ca}, \mathrm{L}}=2.3\left(\mathrm{mS} / \mathrm{cm}^{2}\right)$

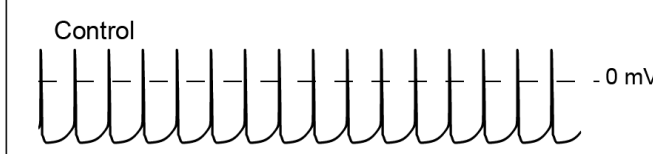

L-type calcium channel inhibition

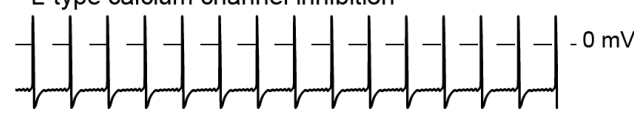

Sodium channel inhibition

$------------.0 \mathrm{mV}$

MWWWWWWWW

Observation similar to the one of

(Guzman et al., 2009)(Seutin et al., unpublished)
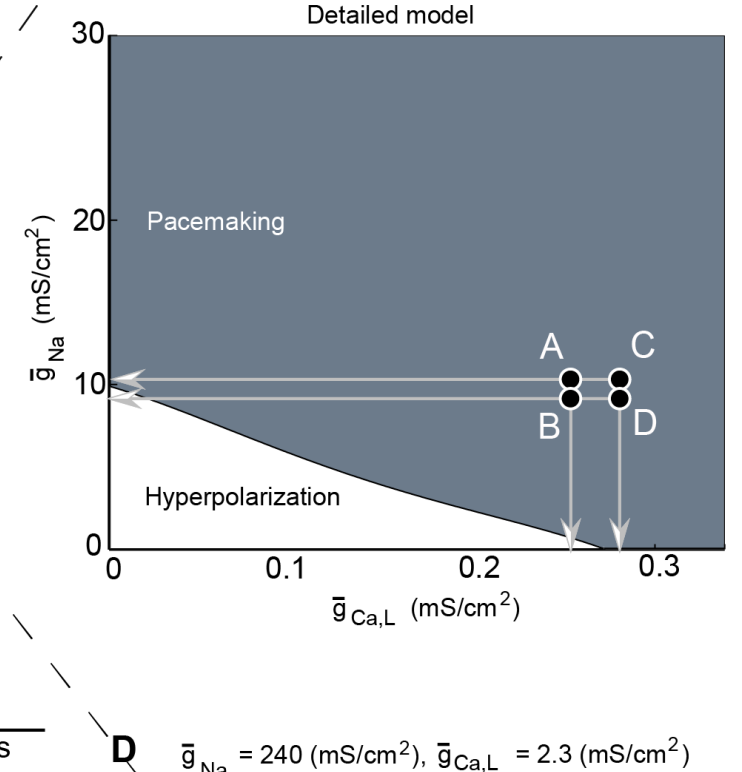

D $\overline{\mathrm{g}}_{\mathrm{Na}}=240\left(\mathrm{mS} / \mathrm{cm}^{2}\right), \overline{\mathrm{g}}_{\mathrm{Ca}, \mathrm{L}}=2.3\left(\mathrm{mS} / \mathrm{cm}^{2}\right)$

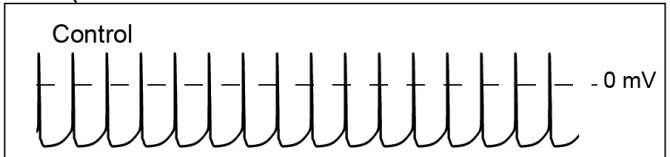

L-type calcium channel inhibition

$-------------0 \mathrm{mV}$

Sodium channel inhibition

$-------------.0 \mathrm{mV}$

MWWWWWMWM

Observation similar to the one of

(Nedergaard et al., 1993)(Puopolo et al., 2009)

(Chan et al., 2007)(Mercuri et al., 1994)(Putzier et al., 2009)

Fig. 14. Cooperation between sodium and calcium channels in the generation of DA neuron pacemaking. The center panel show the type of pacemaker activity according to the value of sodium and L-type calcium conductances in the minimal and quantitative models. The white zone represents hyperpolarized states and the dark blue zone accounts for pacemaking. Each insert shows the behavior of the model in control condition and during a blockade of L-type calcium channels or sodium channels for a particular set of conductances. Taken from [10]. 
space to functional parameters. Without this mapping, the sensitivity analysis in the physiological parameter space can be both fragile and intractable.

\section{Past successes of sensitivity analysis}

The conclusion of our anecdote is that sensitivity analysis of neuronal behaviors is a question of central importance in neurophysiology but that the existing approaches -whether experimental, computational, or mathematical- all suffer from limitations that make progress slow and limited, even in an area where modeling principles are broadly accepted and a lot of experimental data have been accumulated. This conclusion does not imply that one should regard the sensitivity analysis of neuronal behaviors as a hopeless problem. Sensitivity analysis is at the root of many successes of control theory. It is the intractability of the sensitivity analysis question in the repeater problem [3] that led Bode and colleagues to develop the highly successful frequency analysis methods of control theory. A local sensitivity analysis of the loop gain is a highly successful tool for the analysis of linear feedback systems: the superposition principle makes it possible to study the sensitivity analysis of the feedback system at a given frequency. Feedback control then becomes regarded as loop shaping [3], that is, the robustness and performance requirements of the closed-loop behavior are translated as shaping requirements for the sensitivity of the loop gain at different frequencies. Another success of sensitivity analysis is in metabolic control analysis [11][21], where quantitative control coefficients are attached to each elementary reaction of a complex metabolic reaction scheme. In this example, local sensitivity analysis is successful in identifying the limiting steps of a complex nonlinear biochemical process but the analysis is static and its success relies on monotonicity or/and feedforward assumptions about the biochemical pathway.

Excitable behaviors are both dynamic and nonlinear. As such, they violate both the superposition principle of linear feedback systems and the static and/or feedforward assumptions of metabolic pathways. But the modeling principles of conductance-based models suggest that they nevertheless share properties of those two classes of models. The circuit model of a neuron suggests that the capacitor voltage is regulated by many parallel feedback loops. The feedback system is highly nonlinear because conductances are voltage dependent, but this does not preclude a superposition principle between ionic conductances that activate in very different temporal and range windows. The view of each ionic conductance as shaping the loop gain of the circuit in a specific voltage range and in a specific frequency range is not far from the loop shaping paradigm of control theory. Likewise, both the parallel architecture and the biochemical modeling principles of the ionic conductances are highly reminiscent of metabolic pathways. The view of attaching a control coefficient to each activation or inactivation path is not far from the methodology of metabolic control analysis. The dynamic input conductance methodology introduced in [9] is an attempt to replicate earlier successes of sensitivity analysis in conductance-based models.

\section{Voltage-clamp protocol}

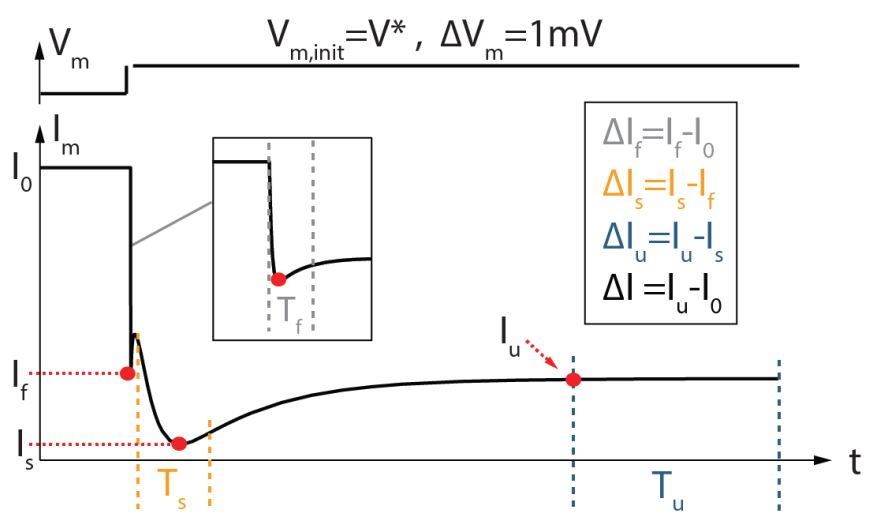

Fig. 15. Example of an experimental measurement of dynamic input conductances in voltage-clamp. A step of potential $\Delta V$ (top) induces variations in the transmembrane current $\Delta I$ (bottom).

\section{DYNAMIC INPUT CONDUCTANCES}

As a one-port resistive circuit, the behavior of a single neuron is entirely determined by its local conductance $g(V, t)$, which is however voltage dependent and time-varying. But one can eliminate the time-varying nature of the conductances by relying on the distinct time scales of any neuronal behavior. A bursting neuronal model has for instance three distinct time scales. This suggests to model the circuit with three independent time-invariant resistive branches, one for each representative time-scale. The conductance of each branch can be determined by mimicking the voltage-clamped experiment of Hodgkin and Huxley: the current variation $\Delta I$ generated by the step of membrane potential $\Delta V$ is decomposed as the sum of three distinct components (Fig. 15)

$$
\Delta I=(\Delta I)_{f}+(\Delta I)_{s}+(\Delta I)_{u}
$$

where $(\Delta I)_{f},(\Delta I)_{s}$ and $(\Delta I)_{u}$ are the fast, slow and ultraslow components, respectively. Each component obeys the sensitivity relationship

$$
\Delta I=\left(-\frac{\partial I}{\partial V}\right) \Delta V=-g(V) \Delta V
$$

where the term $g(V)=\left(\frac{\partial I}{\partial V}\right)$ shapes the sensitivity of the transmembrane current to membrane potential variations. This leads to the decomposition

$$
-g(V) \Delta V=-g_{f}(V) \Delta V-g_{s}(V) \Delta V-g_{u}(V) \Delta V
$$

which gives

$$
g(V)=g_{f}(V)+g_{s}(V)+g_{u}(V) .
$$

The quantities $g_{f}(V), g_{s}(V)$ and $g_{u}(V)$ are called dynamic input conductances. They represent the aggregation of all ionic currents in one specific time-scale.

They can be measured experimentally or computed from the variational analysis of an arbitrarily detailed conductance-based model. In a realistic conductance-based 
model, gating variables exhibit a continuum of voltagedependent timescales. Therefore, a given physiological gating variable can, in principle, contribute to several timescales. The dynamic input conductance in each timescale is therefore expressed as a (voltage-dependent) linear combination of all ionic conductances [9]. For a model composed of the gating variables $X_{n}$, it gives

$$
g_{f, s, u}(V)=\sum_{n} w_{f, s, u}^{X_{n}} \frac{\partial I}{\partial X_{n}} \frac{\partial X_{n, \infty}}{\partial V}
$$

where $w_{f, s, u}^{X_{n}}$ is the contribution of the variable $X_{n}$ in the fast, slow and ulstraslow timescales, respectively. The three dynamic input conductance $g_{f}(V), g_{s}(V)$ and $g_{u}(V)$ can then be interpreted as aggregate conductances in each of the three timescales defining neuronal activity.

Fig. 16, left shows the dynamic input conductances of a bursting neuron model. These voltage-dependent curves make the link between complex conductance-based models and the dynamical motif of neuronal excitability (Fig. 16). The fast dynamic input conductance is mostly positive. It shapes the fast positive feedback responsible for spike upstroke. The slow dynamic input conductance is mostly negative at suprathreshold potential. It shapes the slow negative feedback responsible for spike downstroke. In addition, the slow dynamic input conductance exhibits a region of positive value at hyperpolarized potentials. This slow positive feedback is an essential component of the slow excitability that underlies bursting, as shown above. Finally, the ultraslow dynamic input conductance is mostly negative. It shapes the ultraslow negative feedback responsible for spike frequency adaptation and burst termination. We recover here the motif of two-level excitability (see Fig. 11)

The significance of the dynamic conductances is that they provide a bridge between the qualitative properties of the behavior, which can be determined by a low-dimensional dynamical system organized by a singularity, and the sensitivity analysis of a quantitative physiological model of the conductances, which requires a high-dimensional reaction network. Thanks to this bridge, the biologically relevant question of analyzing how ion channels shape neuronal excitability can be systematically addressed through a sensitivity analysis of the dynamic input conductances with respect to maximal conductance parameters (i.e., density of a particular channel). Computing sensitivity curves of the type $\partial g_{f, s, u}(V) / \partial \bar{g}_{x}$, which evaluates at each membrane potential the derivative of a given dynamic input conductance $g_{f, s, u}(V)$ with respect to a given maximal conductance parameter $\bar{g}_{x}$, one can predict the effect of ion channel variations on neuronal excitability. The dynamical role of an ion channel type is determined by its regenerative/restorative properties in the different timescales of neuronal spiking.

Dynamic input conductances provide the important insight of how ion channels combine to generate or maintain a specific neuronal behavior. This insight is relevant for the quantification of robustness and homeostatic mechanisms that govern neuronal spiking. As an illustration, Fig. 17
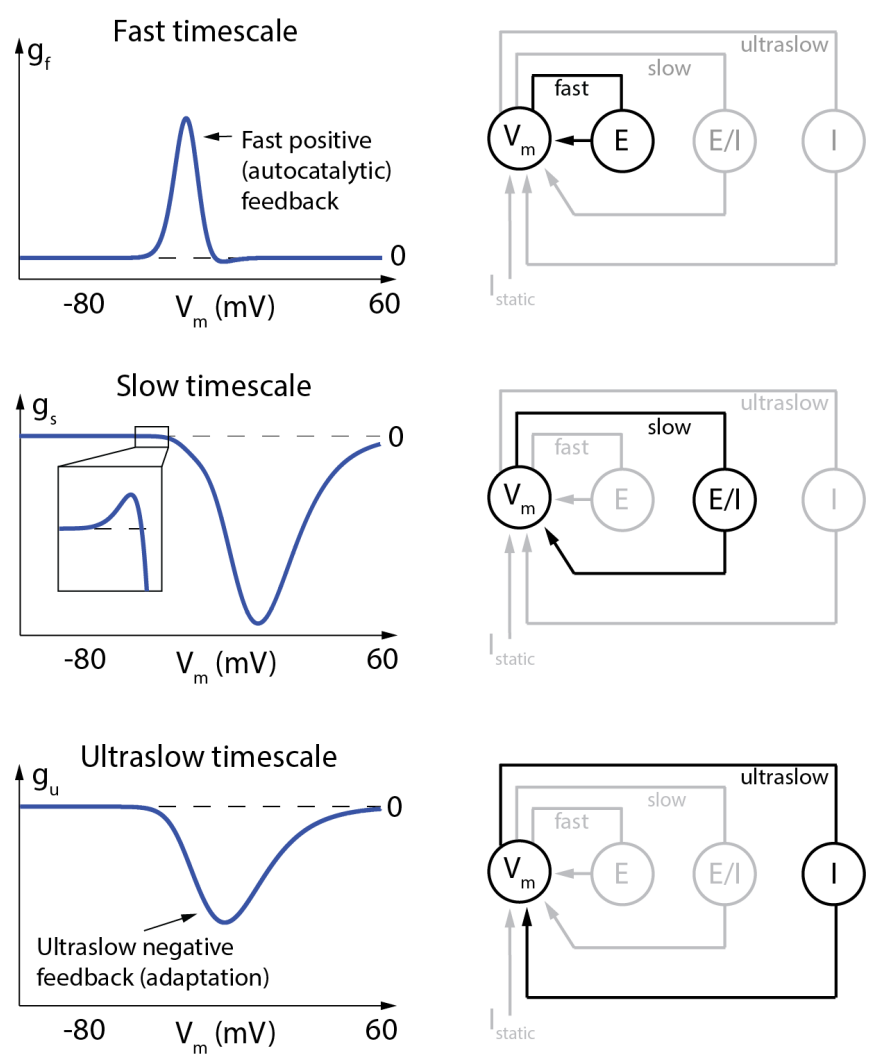

Fig. 16. The dynamic input conductances make the link between complex conductance-based models and the dynamical motif of neuronal excitability. Left, fast, slow and ultraslow dynamic input conductances of a bursting neuron model (from top to bottom). Right, dynamical motif of neuronal excitability.)

shows how the firing activity of a model neuron evolves when the density of a specific ion channel type is increased. When the ion channel density is increased alone, the firing activity is strongly affected. This is because modifying the density of a single channel type can strongly affect the value of one or several dynamic input conductances, which govern the modulation of neuron excitability. On the other hand, the variation of one particular channel density can be compensated for by covarying the densities of other channel types in order to maintain the value of the dynamic input conductances unchanged, as shown at the bottom of Fig. 17. This property, which relies on the fact that many ion channels shape cooperatively the value of the dynamic input conductances, is the basis for the robustness of neuronal activity against the high variability observed in ion channel densities.

\section{HOMEOSTASIS, REGULATION AND DYSREGULATION}

\section{A. Neurons solve a complex regulation problem}

The preceding sections provide two important messages:

1 Electrophysiological properties of neurons arise from a complex interaction between nonlinear components, most notably ion channels and receptors.

2 There are many kinds of ion channels and receptors simultaneously expressed by neurons, many of which 


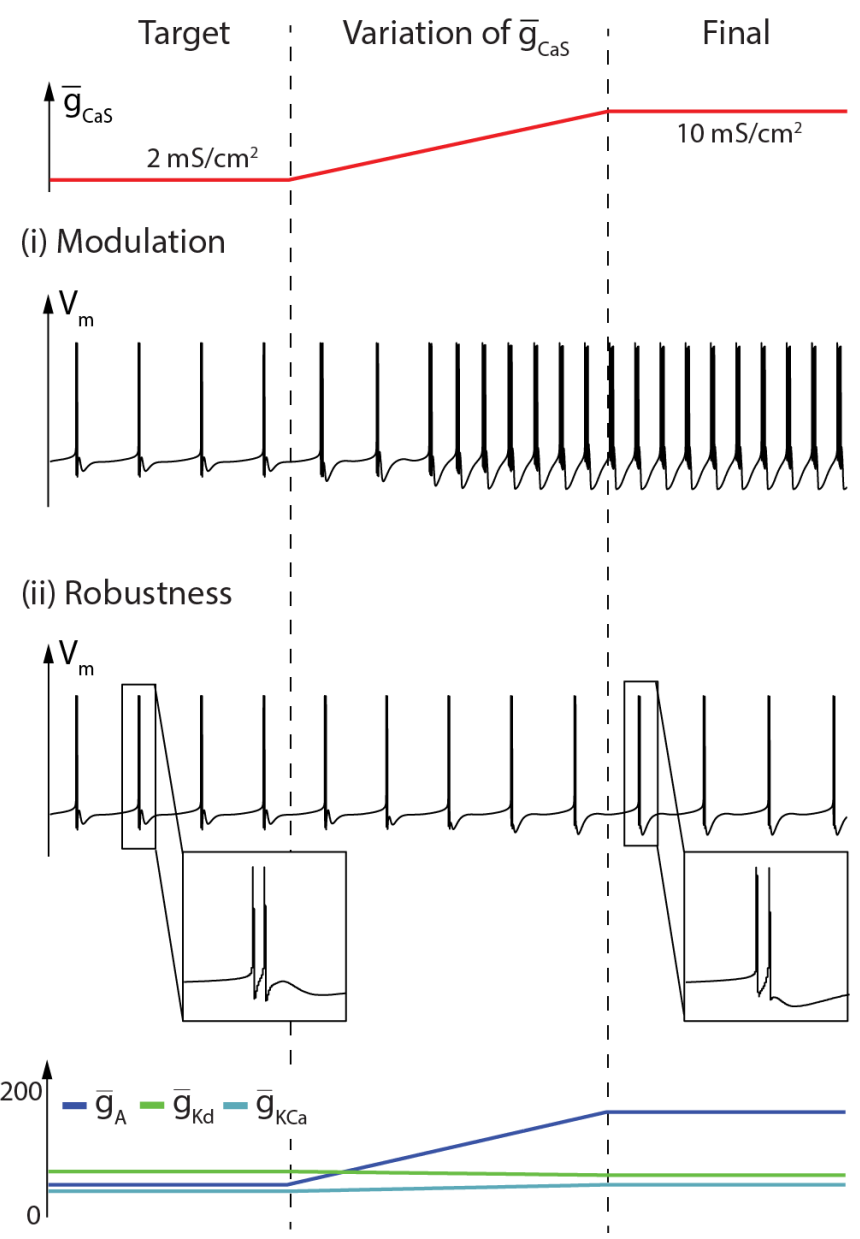

Fig. 17. Compensation mechanism derived from the sensitivity analysis of the dynamic input conductances. Variation of a calcium channel density (top trace) and membrane potential variation over time of a neuron conductance-based model in the absence and the presence of the compensation mechanism (center traces), and variations of channel densities involved in the compensation mechanism (bottom trace).

overlap in their biophysical properties.

The first message tells us that for a neural circuit to work properly, the signaling components in neurons need to be appropriately regulated. Appropriate kinds of ion channels and receptors need to be synthesized and expressed in a neuron at appropriate levels. Achieving the right balance of expression levels is a non-trivial task owing to a complex relationship between the mixture of ion channels expressed in a neuron and its emergent electrophysiological properties at the single cell level, and at the level of a neural circuit.

In respect of the second message, we might be left wondering why evolution has produced a seemingly redundant and unduly numerous array of signaling components. For example, the human genome contains 40 known voltagegated potassium channel genes. Each gene typically encodes multiple versions of a protein subunit that is in turn combined with other subunits to make ion channels, resulting in a combinatorial explosion in the number of kinds of channels that can be expressed. The biophysical properties (such as voltage dependence, ion selectivity, gating dynamics) of these channels might differ substantially, or somewhat subtly, leaving a large number of available degrees of freedom for controlling neuronal excitability [17]. As we saw in previous sections, the contributions of different ion channel types to essential neural dynamics can overlap substantially, even when the properties of individual ion channels differ, meaning that there are many redundant degrees of freedom for controlling neural excitability in a typical neuron.

Together, these messages raise a key question: how do neurons control the expression of this vast array of signaling components so as to achieve and maintain appropriate electrophysiological properties? This is fundamentally a biological question. In terms of biological details (which ion channels are involved, what the architecture of the regulatory mechanism looks like and which biological signaling processes it uses) the answer will likely depend on the type of neuron, the species of animal and the developmental context (e.g. early development when neurons are growing, or adulthood).

Abstractly, the neuron is faced with a very similar problem as an engineer tasked with understanding how individual conductances affect excitability. For example, if the current threshold for initiating a spike is too low, a circuit might become hyperexcitable, so neurons need to adjust specific conductances to raise the spiking threshold. Evidently, neurons somehow solve this problem because, by and large, they achieve appropriate excitable behavior and maintain it throughout an animal's lifetime in spite of continual environmental and internal perturbations. These perturbations may be due to long-lived fluctuations in sensory stimuli or simply noisiness in the underlying biological hardware that nervous systems are comprised of. However, neurons do not have the luxury of being able to predict the effect of increasing or deceasing the expression of a particular conductance so as to deal with a particular perturbation. Instead, neurons are confined to using internal biochemical signals are proxies for sensing their own activity and a set of evolved and perhaps relatively inflexible feedback rules for adjusting ion channel expression to maintain stable function.

\section{B. Feedback control of neuronal excitability}

In addition for compensating for perturbations, neurons must employ feedback control due to the very nature of cellular metabolism. The lifetime of a membrane-bound protein such as an ion channel is many orders of magnitude shorter than the lifetime of the neuron in which it is expressed, the latter being equal to the lifespan of the organism as a rule of thumb. For example, AMPA receptors at excitatory synapses in the mammalian brain are inserted into the membrane, removed and degraded over the course of tens of minutes [24]. Thus, in order for a neuron to maintain its signaling properties, there needs to be constant monitoring and replenishment of ion channels and receptors at an appropriate rate. This observation hints at the existence of feedback control mechanisms that regulate neuronal excitability by controlling the synthesis and degradation rates of ion channels and receptors. In fact, the existence of such a 
feedback mechanism was hypothesized before it was shown experimentally [29].

control

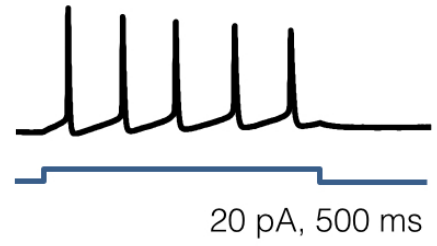

\section{0 hours of depolarisation}

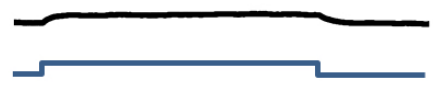

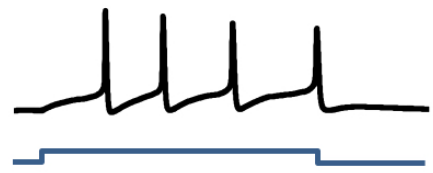

Fig. 18. Experimental evidence of feedback control in neurons. Top: spiking initiated with depolarizing current in a mammalian neuron grown in culture. Middle: no spiking initiated for the same magnitude of depolarizing current as (Top) applied to a neuron grown in chronic depolarizing conditions for a prolonged period (60 hours). Bottom: blocking calcium influx via L-type calcium channels ablates the drop in excitability caused by chronic depolarization. Data reproduced from [31]

Several key experiments [32], [42], [18], [6] have uncovered slow regulatory mechanisms that adjust the expression levels of ion channels and receptors in neurons in response to gross perturbations in electrophysiological activity levels. 'Slow' in this context means a timescale of hours or days - far slower than typical fluctuations in membrane potential activity such as action potentials and network oscillations that are associated with signaling in the nervous system.

An experimental paradigm that we have used for revealing this feedback control mechanism is shown in Figure 18. We exposed neurons to elevated excitatory drive by manipulating the extracellular ion balance, resulting in prolonged depolarization by several millivolts [31], [32]. Over the course of hours to days, the intrinsic firing threshold of the neurons adjusted, making the neurons less excitable. This compensatory, negative feedback mechanism was blocked pharmacologically using L-type calcium channel inhibitors, suggesting that calcium influx is a key feedback signal.

It is important to note that the shift in excitability seen in Figure 18 results from a coordinated change in the expression of multiple ion channel types: sodium currents, potassium currents and transient calcium currents were all found to be altered by the depolarizing stimulus used in these experiments. Therefore, it is possible that a relatively simple signal (net calcium influx) is used by neurons as the control signal for the expression of multiple ion channel types.

A wealth of experimental literature identifies intracellular calcium as a key signal for controlling the expression of
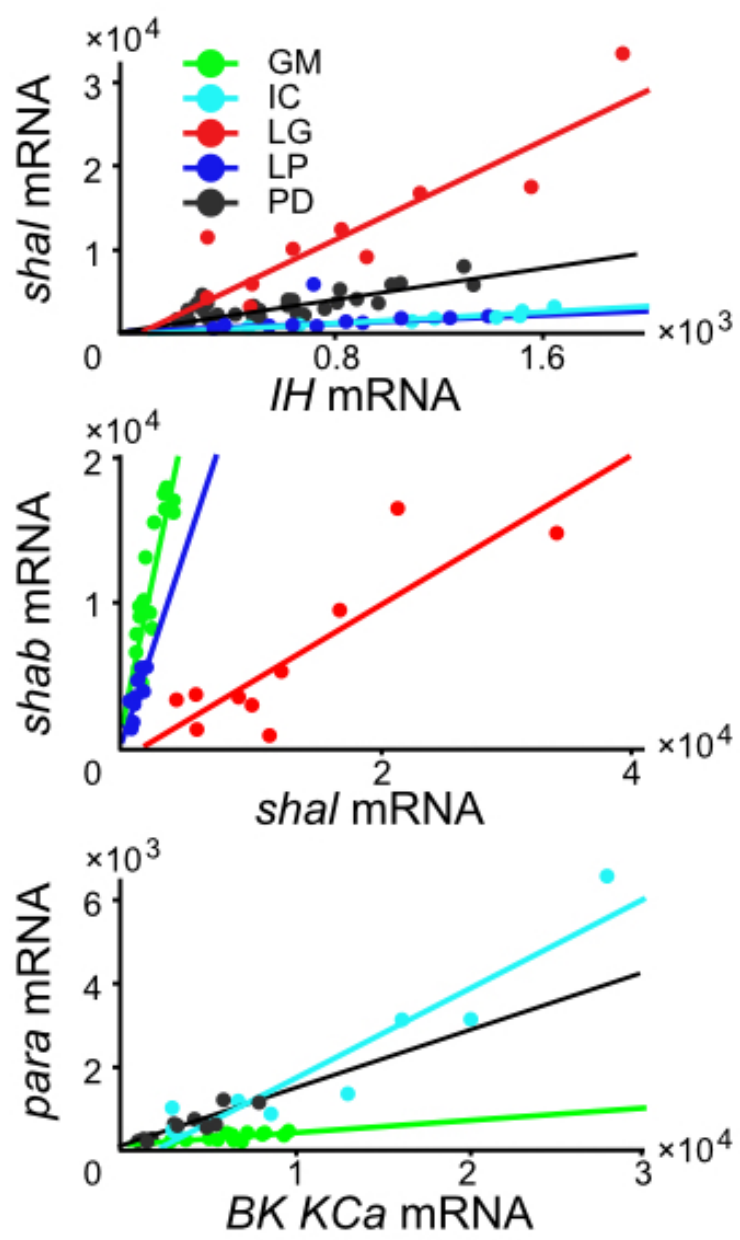

Fig. 19. High variability and correlations in ion channel expression in identified neurons. Gene expression levels (mRNA counts) for six different voltage-gated ion channel genes (para: sodium channel, shab, shal: potassium channels, IH: hyperpolarization activated mixed cation channel, BK KCa: calcium-dependent potassium channel) in identified neurons in the crab Stomatogastric Ganglion (GM: gastric mill neuron, IC: inferior cardiac neuron, LG: lateral gastric neuron, LP: lateral pyloric neuron, PD: pyloric dilator neuron), Axes represent mRNA counts, determined by single cell real time PCR for ion channel transcripts, each data point is a single cell. Data reproduced from [38].

many genes in neurons, including those that code for ion channels [13], [28]. The task of experimentally identifying the regulatory control mechanisms at a molecular level is far from straightforward and it is therefore not feasible to construct literal, detailed models of the biochemical pathways involved. However, there are several key phenomenological features of ion channel and receptor regulation that have been identified, and permit loosely mechanistic, conceptual models to be constructed and analysed [26], [27]. We discuss one such model in what follows.

\section{Neuronal feedback control mechanisms tolerate variabil- ity in ion channel expression levels}

A striking feature of the steady-state expression of ion channel genes is a high degree of variability among neurons 
belonging to the same genetic, physiological and anatomical class. In spite of this variability, these neurons exhibit functionally similar properties, suggesting that the regulatory processes controlling ion channel expression tolerate sloppiness in absolute expression levels and use a coarse regulatory feedback signal. This can be seen in ensemble measurements of the expression levels of different ion channel genes in single, identified neurons [38]. Figure 19 shows three pairwise plots of the expression levels (abundance of mRNA molecules) for six important ion channel genes in five different identified neuron types in a crab motor ganglion. Several-fold variation in mRNA abundance is seen, along with strong, linear correlations between many of the channel genes.

D. Simple models of ion channel regulation reconciles variability and homeostasis

We have seen that neurons employ feedback control to regulate ion channel expression on a very slow timescale and that this feedback control mechanism appears to use calcium influx as a feedback signal. We have also seen that the expression levels of multiple ion channels in single neurons can vary substantially and tends to be correlated. This suggests that neurons do not necessarily care about absolute expression levels of ion channels and instead ensure that relative expression levels are maintained. It is therefore plausible that a simple, scalar signal might be used as an error signal to detect deviation from some target activity level [33].

As a consequence, we may propose a simple feedback control model where calcium influx is integrated by intracellular signaling pathways that control gene expression [34]. Regulation is split into two steps as shown in Figure 20A: calcium concentration, $\left[\mathrm{Ca}^{2+}\right]$, activates (or deactivates) the expression of mRNA molecules, $m_{i}$ for different ion channel types, $g_{i}$. The rates of synthesis and degradation of each of these molecules is assumed to be fixed within each cell type (denoted by the $\alpha$ 's and $\beta$ 's in the Figure) and can be lumped together to give single characteristic time-constants, $\tau_{i}$. This results in a simple set of ordinary differential equations:

$$
\begin{aligned}
\tau_{i} \dot{m}_{i} & =\mathrm{Ca}_{\text {target }}-\left[\mathrm{Ca}^{2+}\right] \\
\tau \dot{g}_{i} & =m_{i}-g_{i}
\end{aligned}
$$

Here, $\tau_{i}$ is the characteristic rate of production of each ion channel mRNA, while $\tau$ is the rate of production of channel protein (which, for simplicity, is assumed to be equal across channel types). In keeping with experimental observations these time-constants are taken to be much longer that the dynamics of membrane potential fluctuations due to channel gating. $\mathrm{Ca}_{\text {target }}$ is a putative equilibrium 'target' calcium concentration that the system maintains. When combined with the membrane equation and the various state equations for Hodgkin-Huxley type conductances, this forms an integral controller, with calcium as the feedback signal (Figure 20B).

An immediate result is that this model produces correlated steady-state conductance densities. Solving system (27) for arbitrary, but small, initial values for the conductances we
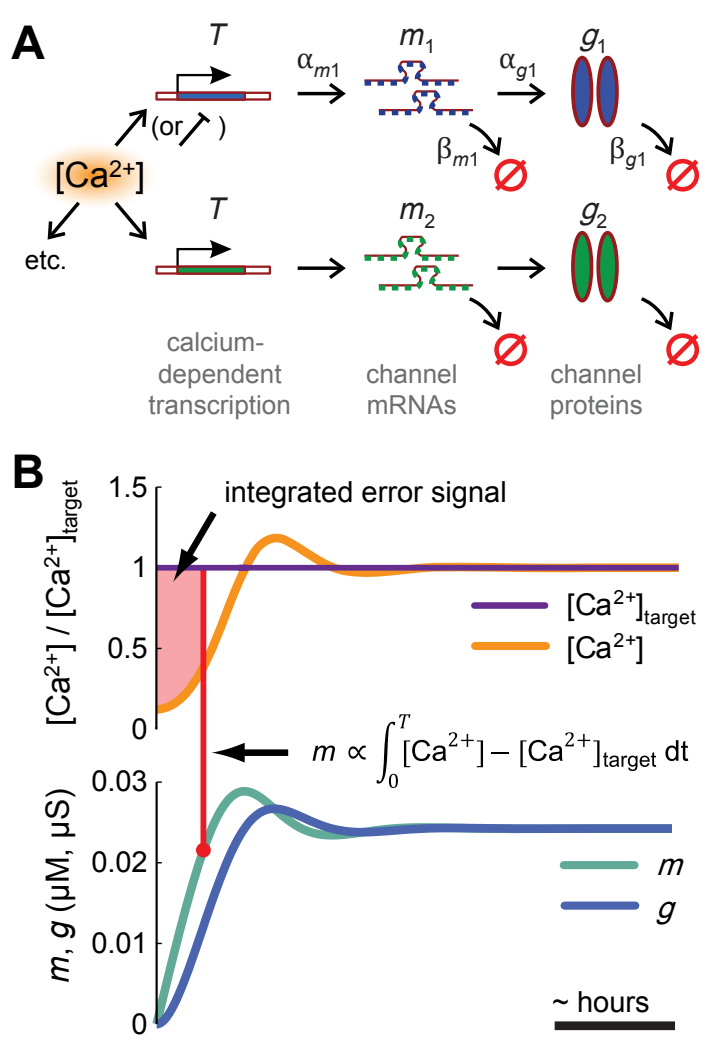

Fig. 20. A simple integral control model of ion channel expression. A Intracellular calcium concentration, $\left[\mathrm{Ca}^{2+}\right]$, determines the rates of production of ion channel mRNAs, which in turn determine the rate of production of ion channel. Each mRNA and ion channel type has constitutive degradation rates. The rates of synthesis $\left(\alpha_{x}\right)$ and degradation $\left(\beta_{x}\right)$ of these molecules are assumed to be characteristic of a particular cell type. B A simplified model of this system constitutes an integral control law (27) with $\left[\mathrm{Ca}^{2+}\right]$ as the feedback signal. Figure reproduced from [34]

find that at steady-state, the ratio of conductance densities is approximately constant:

$$
\frac{g_{i}}{g_{j}} \approx \frac{\tau_{j}}{\tau_{i}}
$$

Not only does a constant-ratio solution resemble the linearly-correlated conductance distributions observed in Figure 19, it also ensures approximately consistent physiological properties when one considers the limit where membrane current dominates the capacitive current [34].

It is not clear, experimentally, whether (27) serves as a model of regulation dynamics that can be taken literally, that is, whether one can really describe channel expression dynamics with two effective time-constants and a single feedback signal. It seems unlikely that a cell as sophisticated as a neuron would use such a crude feedback system to control physiological processes that are crucial for an organism's survival. However, the fact that highly variable levels of ion channel expression are seen suggests that neurons, as feedback systems, are underactuated to some extent. In this context, 'underactuated' is a lose term that simply means 
many variables are controlled by few feedback signals. The simple model presented here captures the extreme case in which only a single scalar feedback signal is used to control the densities of many ion channel types.

Nonetheless, this simple feedback system is able to produce consistent physiological properties in model neurons with a complex set of voltage-gated conductances and is robust enough to generate specific neural circuit activity [34]. Moreover, degeneracy among the properties of the ion channels in the models means that the system is tolerant to loss of a particular ion channel type in many cases. This is shown in Figure 21, where we see that in some situations the simple feedback control model can compensate for deletions of a specific conductance and (approximately) recover its previous behaviour on a long timescale. On the other hand, the model converges to an aberrant state when other, key conductances are deleted, as is seen in Figure 21B, where 'compensation' results in the neuron changing from periodic bursting activity to tonic spiking.

This situation is reminiscent of countless experiments in neuroscience, where sometimes a manipulation (such as deletion of an ion channel gene) is compensated for. Compensation of this kind is seldom perfect, but owing to other sources of robustness in nervous system function, approximate compensation is often good enough. On the other hand, some deletions, physiological insults and disease states cannot be compensated for by the regulatory control systems in biological nervous systems, even when there are potential solutions in parameter space to permit compensation. This is consistent with the idea that the regulatory processes that control ion channel expression are underactuated.

Underactuation means that a number of interesting pathologies can occur. In the context of the simple model above, genetic deletion, or pharmacological ablation of an ion channel type alters the relationship between the feedback signal (calcium) and the expression levels of the remaining conductances. As a consequence, a perturbation that would be innocuous in the absence of the slow feedback control given by (27) can be accompanied by a pathological compensatory response when the system reaches steady-state. This is seen in Figure 21C, where the initial perturbation causes a subtle change in the behaviour of the neuron. However, compensation due to the regulatory control system (27) disrupts spiking activity in the neuron. We speculate that this particularly insidious kind of pathology - one characterized by aberrant compensatory actions of the regulatory systems present in all neurons - constitutes a potential mechanism underlying nervous system disorders and disease states.

\section{CONCLUSION}

Neurons are highly specialized and diverse signaling machines. They derive their electrophysiological properties from a complex interplay of molecular components, most notably ion channels and receptors. We have described the importance of separating the electrical behavior, which can be described by a few nonlinear resistive branches, one for each

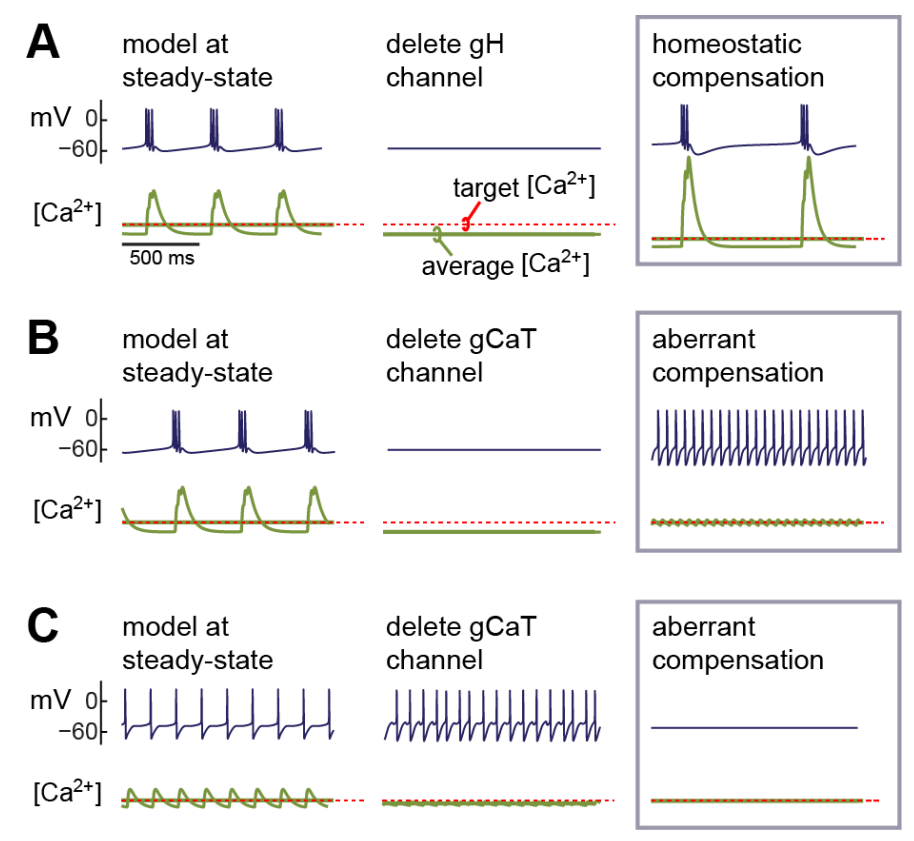

Fig. 21. Homeostatic and aberrant compensation in a simple model of ion channel regulation. A Membrane potential activity (top traces) and intracellular calcium concentration (bottom traces) in a feedback-regulated model neuron (using equations 27). The target value of the control variable, $\mathrm{Ca}_{\text {target }}$, is indicated in red. The left traces show the model at steady state, prior to a perturbation involving deletion of the $I_{H}$-conductance, $g_{H}$ (center traces). Following relaxation to steady-state (right traces), the perturbed model recovers periodic bursting activity. B The same model as (A) with a calcium conductance, $g_{C a T}$, deleted instead of $g_{H}$. In this case, the model does not recover periodic bursting activity and instead fires tonically. $\mathbf{C}$ The same feedback control model as in (A) and (B), but with different regulation parameters which lead to tonic spiking at steady-state. Deletion of $g_{C a T}$ leaves tonic spiking intact, but alters the frequency. Compensation by the regulatory control system following $g_{C a T}$ deletion leads to loss of tonic spiking in this case. Figure adapted from [34]

representative time scale, acting as voltage-dependent positive or negative feedbacks, from the biochemical behavior, which quantifies the contribution of each type of ion channel in a particular frequency range and voltage range. The biophysical modeling principles proposed by Hodgkin and Huxley provide a bridge between the electrical behavior and the biochemical behavior. The electrical behavior is highly nonlinear but it can be described with few variables and it can be analyzed through a local analysis around organizing singularities. The biochemical behavior has a highly parallel and feedforward structure, which can be exploited to draw conclusions from a local parameter sensitivity analysis.

Like most biological systems, the relevant timescales for understanding neuronal activity span many orders of magnitude, up to and including timescales that correspond to the lifetime of the signaling components themselves. Beyond the millisecond-second timescales of spikes, bursts and network oscillations, we explored dynamics of ion channels on the longest known timescale - the timescale over which channels are synthesized and degraded. Again, feedback control is evident but there are numerous unanswered questions concerning the nature of this so-called homeostatic regulation that ensures nervous system stability over the lifetime of 
an organism. Key questions surround the possible modes of failure of homeostatic regulatory control as well as its capacity to autonomously assemble functional circuits from subcellular activity-dependent feedback rules.

The tutorial nature of the paper led us to focus on the internal regulation mechanisms of a single cell, but the proposed methodology is applicable to arbitrary neuronal networks. In that sense, neuronal circuits may provide a rich and novel source of inspiration for the behavioral principles governing the organization of excitable systems.

\section{REFERENCES}

[1] W Ross Ashby. An introduction to cybernetics. 1957.

[2] Corinne Beurrier, Patrice Congar, Bernard Bioulac, and Constance Hammond. Subthalamic nucleus neurons switch from single-spike activity to burst-firing mode. The Journal of neuroscience, 19(2):599609, 1999.

[3] Hendrick W Bode. Relations between attenuation and phase in feedback amplifier design. Bell System Technical Journal, 19(3):421454, 1940.

[4] John H Byrne, Ruth Heidelberger, and M Neal Waxham. From molecules to networks: an introduction to cellular and molecular neuroscience. Academic Press, 2014.

[5] Carmen C Canavier and Richard Spencer Landry. An increase in ampa and a decrease in sk conductance increase burst firing by different mechanisms in a model of a dopamine neuron in vivo. Journal of neurophysiology, 96(5):2549-2563, 2006.

[6] Niraj S Desai, Lana C Rutherford, and Gina G Turrigiano. Plasticity in the intrinsic excitability of cortical pyramidal neurons. Nature neuroscience, 2(6):515-520, 1999.

[7] Guillaume Drion. Regulation of Excitability, Pacemaking, and Bursting: Insights from Dopamine Neuron Electrophysiology. $\mathrm{PhD}$ thesis, University of Liège, 2013.

[8] Guillaume Drion, Maxime Bonjean, Olivier Waroux, Jacqueline Scuvée-Moreau, Jean-François Liégeois, Terrence J Sejnowski, Rodolphe Sepulchre, and Vincent Seutin. M-type channels selectively control bursting in rat dopaminergic neurons. European Journal of Neuroscience, 31(5):827-835, 2010.

[9] Guillaume Drion, Alessio Franci, Julie Dethier, and Rodolphe Sepulchre. Dynamic input conductances shape neuronal spiking. eneuro, 2(2):ENEURO-0031, 2015.

[10] Guillaume Drion, Laurent Massotte, Rodolphe Sepulchre, and Vincent Seutin. How modeling can reconcile apparently discrepant experimental results: the case of pacemaking in dopaminergic neurons. PLoS Comput Biol, 7(5):e1002050, 2011.

[11] David Fell and Athel Cornish-Bowden. Understanding the control of metabolism, volume 2. Portland press London, 1997.

[12] Richard FitzHugh. Impulses and physiological states in theoretical models of nerve membrane. Biophysical journal, 1(6):445, 1961.

[13] Steven W Flavell and Michael E Greenberg. Signaling mechanisms linking neuronal activity to gene expression and plasticity of the nervous system. Annual review of neuroscience, 31:563, 2008.

[14] Alessio Franci, Guillaume Drion, and Rodolphe Sepulchre. Modeling the modulation of neuronal bursting: a singularity theory approach. SIAM Journal on Applied Dynamical Systems, 13(2):798-829, 2014.

[15] Alessio Franci, Guillaume Drion, Vincent Seutin, and Rodolphe Sepulchre. A balance equation determines a switch in neuronal excitability. PLoS Comput Biol, 9(5):e1003040, 2013.

[16] Rafal Goebel, Ricardo G Sanfelice, and Andrew R Teel. Hybrid Dynamical Systems: modeling, stability, and robustness. Princeton University Press, 2012.

[17] Mark S Goldman, Jorge Golowasch, Eve Marder, and LF Abbott. Global structure, robustness, and modulation of neuronal models. The Journal of Neuroscience, 21(14):5229-5238, 2001.

[18] Jorge Golowasch, LF Abbott, and Eve Marder. Activity-dependent regulation of potassium currents in an identified neuron of the stomatogastric ganglion of the crab cancer borealis. Journal of Neuroscience, 19:RC33-1, 1999.
[19] George A Gutman, K George Chandy, Stephan Grissmer, Michel Lazdunski, David Mckinnon, Luis A Pardo, Gail A Robertson, Bernardo Rudy, Michael C Sanguinetti, Walter Stühmer, et al. International union of pharmacology. liii. nomenclature and molecular relationships of voltage-gated potassium channels. Pharmacological reviews, 57(4):473-508, 2005.

[20] Jaime N Guzman, Javier Sánchez-Padilla, C Savio Chan, and D James Surmeier. Robust pacemaking in substantia nigra dopaminergic neurons. The Journal of Neuroscience, 29(35):11011-11019, 2009.

[21] Reinhart Heinrich and Stefan Schuster. The regulation of cellular systems. Springer Science \&amp; Business Media, 2012.

[22] Bertil Hille et al. Ion channels of excitable membranes, volume 507. Sinauer Sunderland, MA, 2001.

[23] Alan L Hodgkin and Andrew F Huxley. A quantitative description of membrane current and its application to conduction and excitation in nerve. The Journal of physiology, 117(4):500-544, 1952.

[24] Qingming Hou, James Gilbert, and Heng-Ye Man. Homeostatic regulation of ampa receptor trafficking and degradation by lightcontrolled single-synaptic activation. Neuron, 72(5):806-818, 2011.

[25] Huifang Ji, Charlotte Hougaard, Kjartan Frisch Herrik, Dorte Strøbæk, Palle Christophersen, and Paul D Shepard. Tuning the excitability of midbrain dopamine neurons by modulating the ca2+ sensitivity of sk channels. European Journal of Neuroscience, 29(9):1883-1895, 2009.

[26] Gwendal LeMasson, Eve Marder, and LF Abbott. Activity-dependent regulation of conductances in model neurons. Science, 259:19151915, 1993.

[27] Zheng Liu, Jorge Golowasch, Eve Marder, and LF Abbott. A model neuron with activity-dependent conductances regulated by multiple calcium sensors. The Journal of Neuroscience, 18(7):2309-2320, 1998.

[28] Huan Ma, Samuel Cohen, Boxing Li, and Richard W Tsien. Exploring the dominant role of cav1 channels in signalling to the nucleus. Bioscience reports, 33(1), 2012.

[29] Eve Marder and Jean-Marc Goaillard. Variability, compensation and homeostasis in neuron and network function. Nature Reviews Neuroscience, 7(7):563-574, 2006.

[30] David A McCormick and Thierry Bal. Sleep and arousal: thalamocortical mechanisms. Annual review of neuroscience, 20(1):185-215, 1997.

[31] Timothy O'Leary. Homeostatic Regulation of Intrinsic Excitability in Hippocampal Neurons. PhD thesis, University of Edinburgh, 2009.

[32] Timothy O'Leary, Mark CW van Rossum, and David JA Wyllie. Homeostasis of intrinsic excitability in hippocampal neurones: dynamics and mechanism of the response to chronic depolarization. The Journal of physiology, 588(1):157-170, 2010.

[33] Timothy O'Leary, Alex H Williams, Jonathan S Caplan, and Eve Marder. Correlations in ion channel expression emerge from homeostatic tuning rules. Proceedings of the National Academy of Sciences, 110(28):E2645-E2654, 2013.

[34] Timothy O'Leary, Alex H Williams, Alessio Franci, and Eve Marder. Cell types, network homeostasis, and pathological compensation from a biologically plausible ion channel expression model. Neuron, 82(4):809-821, 2014.

[35] Astrid A Prinz, Dirk Bucher, and Eve Marder. Similar network activity from disparate circuit parameters. Nature neuroscience, 7(12):13451352, 2004

[36] Ilva Putzier, Paul HM Kullmann, John P Horn, and Edwin S Levitan. Cav1. 3 channel voltage dependence, not ca2+ selectivity, drives pacemaker activity and amplifies bursts in nigral dopamine neurons. The Journal of Neuroscience, 29(49):15414-15419, 2009.

[37] DG Schaeffer and M Golubitsky. Singularities and groups in bifurcation theory. Appl. Math. Sci, 51, 1985.

[38] David J Schulz, Jean-Marc Goaillard, and Eve E Marder. Quantitative expression profiling of identified neurons reveals cell-specific constraints on highly variable levels of gene expression. Proceedings of the National Academy of Sciences, 104(32):13187-13191, 2007.

[39] Rodolphe Sepulchre. Do brains compute? TEDx Talks, 2014.

[40] S Murray Sherman. Tonic and burst firing: dual modes of thalamocortical relay. Trends in neurosciences, 24(2):122-126, 2001.

[41] DG Tucker. The history of positive feedback: The oscillating audion, the regenerative receiver, and other applications up to around 1923. Radio and Electronic Engineer, 42(2):69-80, 1972.

[42] Gina Turrigiano, LF Abbott, and Eve Marder. Activity-dependent changes in the intrinsic properties of cultured neurons. Science, 264(5161):974-976, 1994. 
[43] Jan C Willems. The behavioral approach to open and interconnected systems. Control Systems, IEEE, 27(6):46-99, 2007. 